\title{
LEGAL NDTICE
}

This bork was prefjared is an accormt of winh sponsored by an ageney of the Luited States Government. Nither the tonited Steter Gisern-

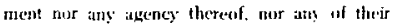
employees, makes any warranty, experess or mo. plied. or asumes any legal liability ur rasponvibilits tor the accuracy. complatemess. ar asefolntess of any information, apposiat us, product, ar process disclosed, or represents that its use wisuld mot infringe prisately insued righis. Heference lerem (1) ally specific commercial product, process, or arvice by trade nisma, trademink, mannfactmes. or otherwise. does an necessurils comstitule or inply its cndorsement, recommendiation. on fin an ing by the L 'nited States (innernment or any anency

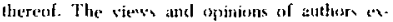
prossed herein do wot neecessitriy state or reflect these of the I nited btates forvernment or att? agency thereof 
$20 \mathrm{~L}-1440 \mathrm{~S}$

DE82 $0205 \% 2$

LBL-14489

Zero-field Spin Relaxation of the Positive Muon in Copper

\author{
Car1 Wil1iam C1awson \\ Nuclear Scietce Division \\ Lawrence Berkeley Laboratory \\ University of Californi: \\ Berkeley, CA 94720
}

Ph.D. Thesis

July 1982

This work was supported by the Director, Office of Energy Research, Division of Nuclear Physics of the Office of High Energy and Nuclear Physics of the U.S. Department oE Energy under Contract DE-ACO3-76SF00098. 
Zero-field Spin Relaxation of the Positive Muon in Copper

by

Car1 W11liam Clawson

\section{ABSTRACT}

The spin relaxation of the $\mu^{+}$in high purity single crystal and polycrystalline copper has teen measured at temperatures between $0.5 \mathrm{~K}$ and $5.2 \mathrm{~K}$ by the zero-field $\mu^{+} \mathrm{SR}$ technique. In both types of sample the experiments show a temperature independent dipolar width $\Delta_{z}=0.389 \pm 0.003 \mu \mathrm{s}^{-1}$ and a hopping rate decreasing from $-0.5 \mu^{-1}$ at $0.5 \mathrm{~K}$ to $-0.05 \mathrm{\mu s}^{-?}$ above $5 \mathrm{~K}$. This $1 \mathrm{~s}$ the first direct proof of a dynamic effect in the low temperature $\mu^{+}$spin relaxation in copper.

The relationship between the zero-field and transverse-fiald dipolar widths is discussed, and the measured zero-field width is found to be $-10 \%$ larger than expected based on the known transversefleld widths.

A new $\mu^{+}$SR spectrometer has been constructed and usad in this work. Tha spectrometer and the associated beam 11nes and data acquisition fasilities are discussed. 
I. INTRODUCTION $\ldots \ldots \ldots \ldots \ldots \ldots \ldots \ldots \ldots \ldots \ldots \ldots \ldots \ldots \ldots \ldots \ldots \ldots \ldots \ldots \ldots$

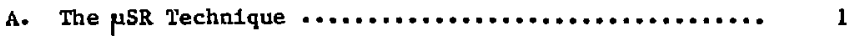

B. History of the Problen ....................... 3

II. EXPERIMENTAL TECHNIQUES $\ldots \ldots \ldots \ldots \ldots \ldots \ldots \ldots \ldots \ldots \ldots \ldots \ldots \ldots . \ldots$

A. Spectrometer and Beam Lines .................... 9

1. $\mu^{+}$Production and Transport ................ 9

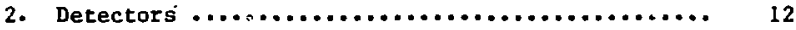

3. Magnetic Flelds $\ldots \ldots \ldots \ldots \ldots \ldots \ldots \ldots \ldots \ldots \ldots \ldots \ldots \ldots . \ldots 14$

4. D. C. Separator .......................... 15

B. Data Acquisition $\ldots \ldots \ldots \ldots \ldots \ldots \ldots \ldots \ldots \ldots \ldots \ldots \ldots \ldots \ldots$

c. Cryogenflcs $\ldots \ldots \ldots \ldots \ldots \ldots \ldots \ldots \ldots \ldots \ldots \ldots \ldots \ldots \ldots \ldots \ldots \ldots \ldots . . \ldots 20$

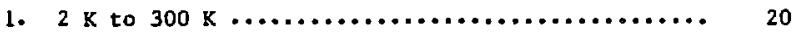

2. Below 2 K $\ldots \ldots \ldots \ldots \ldots \ldots \ldots \ldots \ldots \ldots \ldots \ldots \ldots \ldots \ldots \ldots . \ldots . \ldots$

D. Data Analysts ............................ 22

III. THE DIPOLAR RELAXATION OF THE $\mu^{+} \ldots \ldots \ldots \ldots \ldots \ldots \ldots \ldots \ldots .25$

A. The Static Relaxation $\ldots \ldots \ldots \ldots \ldots \ldots \ldots \ldots \ldots \ldots \ldots \ldots \ldots$

1. Transyerse-field ....................... 27

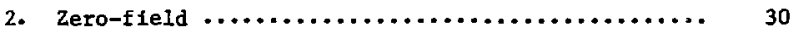

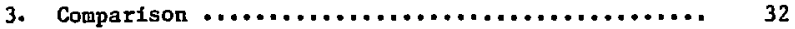

B. Dynamic Effects $\ldots \ldots \ldots \ldots \ldots \ldots \ldots \ldots \ldots \ldots \ldots \ldots \ldots \ldots \ldots \ldots$

c. The Quadrupole Inceraction ................... 36 
IV. EXPERIMENTAL RESULTS $\ldots \ldots \ldots \ldots \ldots \ldots \ldots \ldots \ldots \ldots \ldots \ldots \ldots \ldots \ldots . .40$

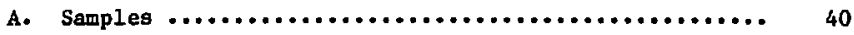

B. Preliminary studies ......................... 40

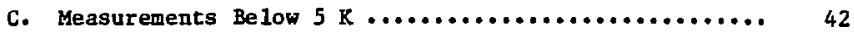

v. DISCUSSION AND SUMMARY $\ldots \ldots \ldots \ldots \ldots \ldots \ldots \ldots \ldots \ldots \ldots \ldots \ldots \ldots \ldots \ldots$

A. The Dipolar Width $\ldots \ldots \ldots \ldots \ldots \ldots \ldots \ldots \ldots \ldots \ldots \ldots \ldots \ldots \ldots$

B. Low Temperature Diffusion and Trapping ........... 51

c. Future Work ............................ 56

Append 1x A: Zero-field Second Moments ................. 58

Acknowledgements $\ldots \ldots \ldots \ldots \ldots \ldots \ldots \ldots \ldots \ldots \ldots \ldots \ldots \ldots \ldots \ldots \ldots \ldots \ldots \ldots . \ldots 1$

References $\ldots \ldots \ldots \ldots \ldots \ldots \ldots \ldots \ldots \ldots \ldots \ldots \ldots \ldots \ldots \ldots \ldots \ldots \ldots \ldots \ldots \ldots$

Tables $\ldots \ldots \ldots \ldots \ldots \ldots \ldots \ldots \ldots \ldots \ldots \ldots \ldots \ldots \ldots \ldots \ldots \ldots \ldots \ldots \ldots \ldots \ldots \ldots . . \ldots$

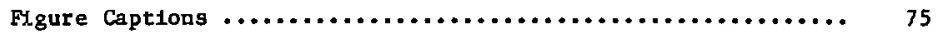

Fugures $\ldots \ldots \ldots \ldots \ldots \ldots \ldots \ldots \ldots \ldots \ldots \ldots \ldots \ldots \ldots \ldots \ldots \ldots \ldots \ldots \ldots \ldots \ldots$ 
CHAPTER I

\section{INTRODUCTION}

\section{A. The HSR Technique}

The work to be presented in this thesis is an experimental study using the techniques of posttive muon $\operatorname{sptn}$ rotation $\left(\mu^{+} \mathrm{SR}\right)$. PolarIzed positive muons $\left(\mu^{+}\right)$are 1mplanted une at a ilme into the experimental sample, and for an ensemble of $-10^{7}$ decay events $\mu^{+} \rightarrow e^{+}+V_{e}+\bar{V}_{\mu}$ the time interval between the arrival of the muon and the detection of the positron $\left(e^{+}\right)$is measured. For each of several $e^{+}$detectors a histogram of the time values is collected. Since the angular distribution of the posttrons is asymmetric with respect to the $\mu^{+}$spin difection, the histogram for a detector can be analyzed to yleld the spin polarization of the $\mu^{+}$ensemble, projected on the detector direction, as a function of time. Several reviews of the subject ${ }^{1-3}$ describe the experimental techniques, and a more detalled discussion will be given in chapter II.

If the $\mu^{+}$is stopped in a magnetic field $H_{0}$ perpendicular to its polarization, 1 ts $\mathrm{spin}$ precesses at the Larmor frequency $w_{0}=\gamma_{\mu} H_{0}$ In the local field $H_{0}$. If the local field consists of the applied fteld plus a random field that varies from one muon to the next, e.g. nuclear dipolar ffelds at different sites in a crystal, the ensemble polarization will decay at a rate proportional to the magnitude of the random ffeld. The signal resulting from this is essentially a 
time domain free Induction decay spectrum--1tg Fourler transform would give the line shape that would be obtalned in a radio-frequency absorption experiment. This constitutes the conventional transverse-fleld $\mu^{+}$SR technlque, which has been the backbone of $\mu^{+} S R$ research for about 10 years.

of great importance in this work is the zero-field $\mu^{+}$SR technique, ${ }^{4}$ pioneered by the University of Tokyo group, wherein no applied magnetic field to present; the $\mu^{+}$feels only the local magnetfc ffelda created by the sample. Here the name "muon spin rotation" is somewhat Inaccurate; we should call it muon spin relaxation 1nstead. The spectrum observed in this case contalns no osc1llations (at least in non-maginetj.c metals), but the relaxation of the d.c. polarization can be observed. The relaxation envelope, however, is not the same as would be seen in transverse field. As we shall see In chapter III, it is especially sensitive to dynamfc relaxation, 1.e., to changes in the local field at the muon during its lifettme. The behavior of the $\mu^{+}$in materials and the regime in which we can make measurements with $\mu^{+}$SR are determined by several of its fundamental properties, which are listed below.

(see next page) 


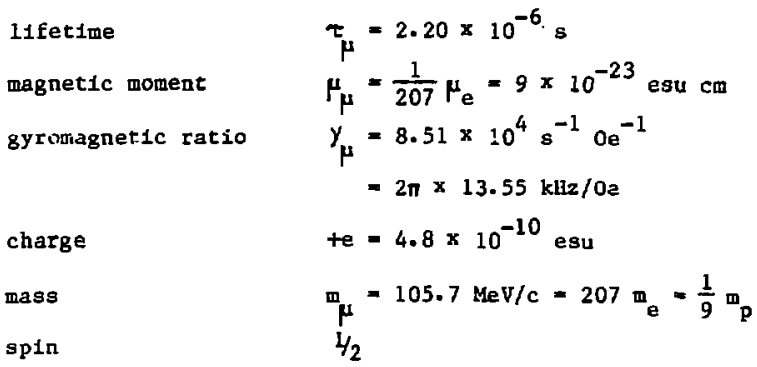

The measurements are limited in time by the finite muon lifetime; magnetic fields that we may be concerned with must be on the order of a gauss or larger.

B. History of the Problem

In 1972, Gurevich et al. 5 reported the first measurements of the $\mu^{+}$spin relaxation in copper. This experiment was done in a magnetic fleld perpendicular to the inttial muon polarization, and the resultIng precession signal exhibited a temperature dependent relaxation rate. This was attributed to dipolar magnetic fields from the neighboring $\mathrm{Cu}$ nuclef, with temperature dependence caused by motional narrowing due to diffusion of the $\mu^{+}$. From data taken between $77 \mathrm{~K}$ and room temperature ( $\left.F H_{\mathrm{g}} .1\right)$ they extracted the $\mu^{+}$hopping frequency $\nu$ and $f$ it it with an Arrhenius law

$$
V(T)=V_{0} e^{-Q / T}
$$

with an activation energy $Q=540 \mathrm{~K}(45 \mathrm{meV})$ and a preexponential factor $V_{0} \equiv 3 \times 10^{7} \mathrm{~s}^{-1}$. This 1mplies that the $\mu^{+}$is essentially stationary below about $80 \mathrm{k}, 1 . e$, the hopplng time at that tempera- 
ture is much longer than the muon lifetime.

The relaxation rate was defined 1ndependently of the shape of the relaxation as the Inverse of the time required for the polarization to decay to $1 /$ e of 1 ts Initial value. However, this parameter was used to compare a Gaussian relaxation function to the $77 \mathrm{~K}$ data with good agreement, Indicating that the $\mu^{+}$is stationary, or nearly so, at this temperature. The relaxation rate was found to be $-0.22 \mu \mathrm{s}^{-1}$ for $\mathrm{T}=77 \mathrm{~K}$.

Further work ${ }^{6}$ extended the temperature range down to $30 \mathrm{~K}$ and showed that the value of the preexponential constant was far smaller than it should be if the diffusion were due to classical over-barrier hopping, and that the activation energy was less than the barrier helght known for hydrogen diffusion. A simple model of tunneling through a rectangular barrier using plausible values for the width and helght was used to explain the results. In this picture, the activation energy $Q$ represents the work aeeded to "prepare" the adjacent site by expanding it to fit the $\mu^{+}$, but the overall barrier height is still fmportant as it determines the tunneling rate. It was also noted that the temperature dependence deviated from the Arrhenius law at the high and low ends of the temperature interval studied.

Pursuing these studies, Caman1, et al. 1nvestigated the orientation, magnetic fleld, and temperature dependences ${ }^{7-10}$ of the transverse relaxation. When combined with the work of Hartmann 11 concerning the quadrupole effect on the dipolar line width, the 
resilts that emerged seemed like a relatively complete and consistent picture of the $\mu^{+}$diffusion in copper: the $\mu^{+}$, trapped at an octahedral (0) Interstitial site in the Cu lattice, diffuses via a thermally activated process glving a spin relaxation well described by the "Abragam formula"12 (which is actually due to Kubo and Tom$1 a^{13}$, using the Van Vleck ${ }^{14}$ value for the dipolar width at high field and a correlation time governed by an Arrhenius law temperature dependence. At lower flelds the dipolar with is changed from the high fleld value due to the precession of the cu nuclear quadrupoles In the electric field gradient that exists around the $\mu^{+}$. The highfield relaxation was explained by assigning the $\mu^{+}$to an octahedral Interstitial site and allowing the nearest-neighbor separation to be Increased by about $5 \%$ due to the expansion of the lattice around the $\mu^{+}$.

To be fair, we should note that the first mention of the approxfmate orfentation independence of the relaxation rate in low flelds was published by Hartmann, et. al. 15

Theoretical support for this model has come from the work of $H$. Teichler. ${ }^{16-20}$ He has calculated the hopping rates of the hydrogen isotopes, Including the $\mu^{+}$, in Cu using the quantum mechanical. diffusion theory of Flynn and Stoneham ${ }^{21}$ and Kagan and Kl1nger. ${ }^{22}$ HIs approach uses non-linear screening to describe the muon-electron interaction and a pseudopotential to represent the screened $\mu^{+}-$ lattice Interaction. With the Born-Oppenheimer approximation, these yleld potential energy curves (F1g. 2) which show a shallow local 
minimum at the tetrahedral (T) site and an absolute minimum at the 0 site.

His conclusion is that the $\mu^{+}$diffuses due to veakly lattice activated tunneling between neighboring octahedral sites. This is in contrast to the case of the 1ootopes H, D, and $T$, for which the tetrahedral site can support metastable bound states, whereas the lighter mass of the $\mu^{+}$causes 1ts zero-point energy to lie above the top of the well. The heavier 1sotopes diffuse $v i a \quad 0 \rightarrow T \rightarrow 0$ phononasslsted over-barrier hopplng whereas the $\mu^{+}$tunnels directly between ground state levels at octahedral sites. Since the energy levels of the $\mu^{+}$are changed by the lattice distortion, the $\mu^{+}$can $t$ tunrel untll a lattlce fluctuation causes a levei colncidence between neighborlng sites. The activation energy for the hopping of this strongly self-trapped "small poiaron" state is then just the lattice deformation energy necessary to equalize the $\mu^{+}$ground state levals at adjacent sites. This explains the small value of $Q$ found in Refs. 5 and 6.

This "tunneling-hopping" theory also explains the deviations from the Arrhenius law seen in the experiments as a result of the zero-poinc fluctuations of the lattice modes as compared to the thermally excitel phonisns.

By 1980 , then, 1 t appeared the the $\mu^{+}$in Cu was well understood. In particular, the $\mu^{+}$seemed completely "frozen" below about $80 \mathrm{~K}$. At that time Hartmann et al. ${ }^{23}$ announced their observation at CERN of a decrease in the $\mu^{+}$transverse-field depolarization rate below $4 \mathrm{~K}$ 
(F1g. 12). They interpreted this result as being due to motional narrowing: the $\mathrm{p}^{+}$mobility increases as $\mathrm{T}$ is reduced below about $4 \mathrm{~K}$.

In 11 hht of the previous knowledge this is a somewhat starting result, and one must consider the alternatives. In perticular one should be careful about inferring motion from changes in the transverse-field relaxation. The reason is that much transversefield work does not discriminate well between static and dynamic relaxation. The difference between static and dynamic processes depends critically on the shape of the relaxation function, not just on the relaxation rate. A static process ylelds a transuerse relaxation function closely resembling a Gaussian (Gaussian IIne shape); whereas a dynamic process, where the field seen by an tndividual muon is not constant throughout the muon's lifetime, gives an exponential relaxation (Lorentzian line shape). 12 This can be understood heuristically by comparing a spin that precesses linearly from its inftial orientation with one performing a random walk.

The shape of the relaxation function was not we 11 measured in the experiments of Ref. 23; they assumed a Gaussian shape and stated that the data showed no clear preference for one shape versus the other. Thus their result is a shape-independent measurement and could be due to the $\mu^{+}$finding a different sice to occupy ar lower temperatures, for instance because of the presence of a high concentration of a shallow trap. The difference in the resultiag nuclear dipole sum sould explatn the reduced depolarizacion rate. 
We should note that in later communications from the CERN group, 24,25 they do mention difficulties with a Gaussian fit at the lowest temperatures; however they still use the Gaussian and make no mention of attempts to use exponential or Abragam relaxation functions. In addition, Ref. 25 preseots data which do indicate that the $\mu^{+}$site is probably octahedral at the lowest temperaturee, although the orientation dependence is weakened, presumably smeared out because of the motion of the muon.

It therefore became desirable to conduct a zero-field experiment In order to help distingulsh among these alternatives. In this work the resuits of such an experiment ${ }^{26}$ are presented. We have shown that the zero-field relaxation clearly indicates that the decrease in the relaxation rate below $5 \mathrm{~K}$ is due to motion of the $\mathrm{\mu}^{+}$.

A principal result of the theory of zero-ficld relaxation is that the polarization approache: $1 / 3$ as $t \rightarrow \infty$ if the local field is time-independent. In our experiments the $\mu^{+}$polarization in zero field recovers nearly completely to an asymptotic value of $1 / 3$ for long times at $5.2 \mathrm{~K}$; below $2 \mathrm{~K}$ the recovery is completely suppressed. $A$ : we shall see in chapter III, the asymptotic polarization of $1 / 3$ is a consequence of only the 1sotropy and static character of the local field distribution, so this constitutes a direct and unambiguous proof of the dynamic nature of the low temperature relaxation. No assumptions about possible locations of the $\mu^{+}$or details about the distribution in magnitude of the local field are required. 
CHAPTER II

EXPERIMENTAL TECHNIQUES

\section{A. Spectrometer and Beam Lines}

The experiments were conducted at the Tri-Unfversities Meson Facility (TRIUMF), operated by University of British Columbia, Univers1ty of Victorla, Simon Fraser University, and University of Alberta at the U.B.C. campus in Vancouver, B.C., Canada. The cyelotron produces an external $500 \mathrm{MeV}$ proton beam with ar Intensity of 30-100 $\mu \mathrm{A}$, which atrikes a pion praduction target, typically a few mm or cm In size, producing coplous quantities of secondary charged particles, primarily pions, muons, and electrons. The secondary particles are collected, momentum-seiected, and delivered in a beam to the experfmenter by magnetic optics.

A. 1. $\mu^{+}$Production and Transport

In a conventional $\mu^{+}$beam, ${ }^{1} \mu^{+}$are collected from the "inflight" decay of $n^{+}$, which gives rise to $\mu^{+}$of typically $100 \mathrm{MeV} / \mathrm{c}$ momenturn. These $\mu^{+}$have a range of order $10 \mathrm{~g} \mathrm{~cm}^{-2}$ in matter. The $\mu^{+}$are slowed in a degrader material (usually polyethylene) and pass through seveial plastic scintilidtion counters into the sample. A counter behind the sampie is used to veto the event if the $\mu^{+}$emerges from the sample, determining if the $\mu^{+}$has stopped in the sample. 
The range spread of the $\mu^{+}$after passing the degrader 1s still sufficient to require the ise of thick ( $\left(21 \mathrm{~g} \mathrm{~cm}^{-2}\right.$ ) rargets in order to stop an apprectable fraction of the incident muons. The $\mu^{+}$are focused to a spot $-3-5 \mathrm{~cm}$ in diameter, so the optimum target size is of order $3 \times 3 \times 3 \mathrm{~cm}^{3}$.

This is a rather sizable piece of material. Many interesting materials in solid state physics are rare or expensive, or for other reasons difficult to obtain in such s1zes. Outside of solid state physics proper, one may wish to stop $\mu^{+}$in gases, powders, or other low-density targets. This is made possible by the use of the "surface muon" beam (also known as che "Artzona" beam) which restits from the decay of pions at rest in the laboratory. 27

When the proton beam is incident on the production target, s1zable numbers of pions are created which do not escape the target. Those that are near the surface of the production target can enlt a muon which escapes the target.

Considering the elementary kinematics of the two-body decay of a spinless particle $\left(n^{+} \rightarrow \mu^{+}+V_{\mu}\right)$, and nocing that the neutrino is left-handed and massless, we have two Important facts in the plon rest frame (laboratory frame):

(1) The $\mu^{+}$is $100 \%$ polarized opposite to its momentum, and

(2) the $\mu^{+}$is monochromatic, $p_{\mu}=29.8 \mathrm{MeV} / \mathrm{c}$ (k1netic energy $\mathrm{T}_{\mu}=$ 4. $1 \mathrm{MeV} / \mathrm{c})$. 
The first point is advantageous; it guarantees us maximum asymmetry in the $\mu^{+}$SR signal, but the second point is the most important property for our purposes. The rarge of the surface $\mu^{+}$is $-150 \mathrm{mg} \mathrm{cm}^{-2}$ with a range width of $-30 \mathrm{mg} \mathrm{cm}{ }^{-2}$ (FIg. 3).

An additional advantage of the surface $\mu^{+}$is seen from consideration of beam optics. A conventional $\mu^{+}$beam is obtained by first selecting pions of a given momentum, allowing them to decay in flight, and tuning the later section of the beam line slightly above or below the mean pion momentum to select muons wich decayed in the forward or backward directlons, respectively, relative to the pion momentum. The decaying pions form an extended source of muons so the beam spot is usually large. Surface muons, however, all emanate from the pion production target, an image of wich is focused on the experfmental target. There is also an advantage in that the solid angle for accepting pions is now $2 \pi$, rather than a few tens of msr. With good optics, beam spots as small as $-1 \mathrm{~cm}$ in dianeter FWH with very sharp edges have been obtained, and a flux of over $10^{6} \mu^{+} / \mathrm{s}$ can be delivered into a $5 \mathrm{~cm}^{2}$ spot. ${ }^{28}$ Thus experiments on samples as smail as $1 \mathrm{~cm} \times 1 \mathrm{~cm} \times 0.5 \mathrm{~mm}$ are posstble, representing a factor of $\geq 10^{2}$ decrease in the total mass of sample needed.

The short range of the surface $\mu^{+}$provfdes another advantage. A $\mathrm{\mu}^{+}$beam is likely to be contaminated with significant numbers of positrons, In some cases as many as 100 for every muon. At i.he higher momenta of conventional muons, both muons and positrons are minfmum-ionizing and hence have similar energy losges in scintilla- 
tion counters. But the energy loss of a $29.8 \mathrm{KeV} / \mathrm{c} \mu^{+} 18$ a factor of -10 above the mintmum-1ontzing value, allowing pulse height discrimination between muons and positrons.

The short range of the surface $\mu^{+}$also creates one primary disadvantage: the $\mu^{+}$beam must be transported from the pion production target to the experimental target, and the amount of lotervening matter mugt be small. For exapple, it 1s possible to fully stop a surface muon beam in about $1 \mathrm{~m}$ of air. It is necessary at the very least to pass the $\mu^{+}$through a scintillation counter, and the enhanced energy loss mentloned In the last paragraph aids in the solution of this by allowing the use of thinner scintillators.

The surface $\mu^{+}$also suffer multiple coulomb scattering in passIng through windows and counters. In the present apparatus the primary source of this is a $0.003^{\prime \prime}$ mylar window $6^{\prime \prime}$ upst.ream from the target, which will cause a mean scattering of $0.15^{\prime \prime}$ at the sample. To estimate the effect of multiple scattering, we make a worst-case assumption that the unscattered beam uniformly fil?s a 0.7 " beam spot. Upon canvolving this with a Gaussian scattering distribution, 1t is found that $-1 \%$ of the bean is stopped outside the $l^{\prime \prime}$ target diameter. This is negligltle for our purposes.

\section{A. 2. Detectors}

To take full advantage of the propertles of surface muons, we have bullt a $\mu^{+}$SR spectrometer $(F 1 g$. 4), dubbed "Eagle", designed specifically to utilize them. The chief constraint to be met is to 
transport the $\mu^{+}$to the target through as little matter as possible. Th1s is achleved by having the entire array of counters as well as the sample sttuated in the same vacuum chamber.

A $0.003^{\prime \prime}$ nylar window 1mmediately upstream from the beam counter is necessary to 1solate the experimental vacuum chamber from the beam line and cyclotren vacuum. The $\mu^{+}$pasa this window and a $0.012^{\prime \prime}$ scintillator (" $D$ " counter) before entering the target. These two items yield a total thickness of $42 \mathrm{mg} \mathrm{cm}^{-2}$. Additional windows, etc., in the target assembly must be thin enough to keep the total thickness upstream of the sample below $100 \mathrm{mg} \mathrm{cm}^{-2}$.

Four positron telescopes consisting of $1 / 4^{\prime \prime}$ thick plastic scintillators (L1-L2, R1-R2, EI-F2, B1-B2) are arranged parallel and perpendicular to the beam direction. The $F$ and $B$ counters are provided with holes in order to pass the $\mu^{+}$beem and, in the case of $F$, to pass any beam $\mathrm{e}^{+}$which penetrate the target. The solid angle subtended by the four counters is about $2 / 3$ of $4 \pi$, and the net efficlency for detecting decay positrons, including dead time, is about $20 \%$.

The D counter has a covering of $0.0003^{\prime \prime}$ aluminized mylar on each side, and the $\mathrm{e}^{+}$counters are covered with $0.00 I^{\prime \prime}$ alumintzed mylar. UVT Lucite light guides from the scintillators exit the bottom of the vacuum chamber through o-ring seals and enter photomultiplier (RCA 8575) assemblies. The light guldes outside the vacuum chamber are covered with aluminum foll and black vinyl wrapplng. The entire scintillator, light gulde, and phototube assembly is mounted on the 
bottom cover plate of the vacuum chamber, which is in turn supported by Acme thread screws and sprockets In order to factlitate entry Into the vacuum chamber.

Between the positron counter telescope elements are placed blocks of graphite to act as positron moderators. This helps co Increase the positron asymmetry by cutting off the low end of the positron energy spectrum ${ }^{1}$ and, more Importantly, helps prevent scattered $29.8 \mathrm{HeV}$ beam positrons from firing an "ie" colncidence, which can be a troublesome source of notse.

Immediately upstream of the beam counter is a $1^{\text {" thick brass }}$ col.timator with tapered holea from $0.2^{\prime \prime}$ to $0.7^{\prime \prime}$ In diameter selectable from outside the chamber. Tis serves to define the beam enterIng the sample. This thickness and position of this collimator are such that positrons from muons stopped in it have very low probab1lity for firing any of the " $e$ " coincidences. Three additional one Inch thick brass collimators of dlameters $1.0^{\prime \prime}, 1.125^{\prime \prime}$, and $1.25^{\prime \prime}$ are placed further upstream to protect the B2 counter from beam particles and to reduce the number of $\mu^{+}$which stop in the final collimator.

The target assemblies are inserted through the top of the vacuum chamber.

\section{A. 3. Magrietic Fields}

A patr of water-cooled Helmholtz colls of mean diameter $22^{\prime \prime}$ surrounding the vacuum chanber is used to provide a transverse (vertical) magnetic field at the sample. Although in principle these mag- 
nets can reach $6.5 \mathrm{kOe}$ the low magnetic rigidity of $29.8 \mathrm{HeV} / \mathrm{c}$ muons prevents the use of flelds above about 1 kOe. The field is controlled by regulating the current through the colls. The current is measured using a resistive shunt and converted to a field with the factor $4.630 \mathrm{e} / \mathrm{A}$, which was measured by a tall probe and by the $\mu^{+} \mathrm{SR}$ precession frequency in aluminum. With this configuration we can measure both transverse components of the polarization with the $e^{+}$ telescope pairs (L-R) and (F-B).

A small pair of air-cooled colls $1 \mathrm{~s}$ used to create longitudinal flelds of up to 10 oe. With no vertical fleld applied this al in:te measurement of the longltudinal polarization in fields from $0-10$ oe with the $F$ and $B$ telescopes. These coils are used primarily for compensating stray longitudinal flelds.

The scintillator and vacuum chamber assembly are constructed of non-magnetic materfals to preserve the field homogenefty and 11near1ty at the sample. For the zero-field runs the stray magnetic field of -1.5 oe at the sample position was measured with Hall probes and reduced to $<0.2$ De with approprlate currents in the two sets of co1ls. This reduces the background precession of the $\mu^{+}$to $\leqslant 1^{\circ} / \mu$ s.

\section{A.4. $\underline{\text { D. }}$. Separator}

The spectrometer has been used successfully in 3 different beam areas of TRIUMF, the M13, M9-F2, and M9-W3 areas, but the experiments described here were performed primarily on the My-W3 beam line due to Its exceptionally clean $\mu^{+}$beam. Th1s is achleved by the use of a 
Wien filter velocity selector, which uses crossed transverse d.c. electric and magnetic flelds to pass only particles of a glven veloc1ty. The $29.8 \mathrm{MeV} / \mathrm{c}$ beam positrons, which are extremely troublesome in a longitudinal measurement, are not selected againat by purely magnetic optics, which pass all particles of a given charge and momentum. A selection by veloctty following momentum selection allows only a single species of particle to reach the experimental area, and we obta1n a beam with undetactably small positron contamination.

Becauge the zero-field $\mu^{+}$SR technique depends critically on the behavior of the polarization at long times where the counting rate is quite low, the rleanliness of the beam is of great ixportance.

\section{ㅂ. Data Acquisition}

The conventional time-differential $\mu^{+}$SR measurement ${ }^{1}$ is used. The signals from the phototubes are discriminated and fed into the system of NIM $\log 1 c$ shown in $F 1 g$. 5, which performs the $\mathrm{e}^{+}$coincidences and rejection of "bad" events. A $1 \mathrm{GHz}$ time digftizer (TRIUMF model B080), or "clock", recelves a start pulse at the time $t_{\boldsymbol{\mu}}$ that the $\mu^{+}$passes the beam counter and a stop at the time $t_{e}$ that an $e^{+}$passes any of the telescopes $L, R, F, B$. A routing bit is sent to a colncidence buffer (Model C212, EG\&G Ortec, Oak Ridge, Tenn. 37830) according to which $e^{+}$telescope fired, and for each telescope a histogram of "good" events vs. $\left(t_{e}-t_{\mu}\right)$ is accumulated 1n the memory of a PDP-11/40 mintcomputer and pertodirally updated on a disk file. The data are written 1nto the PDP-1l memory by a CAMAC system driven by 
an MBD-11 microcomputer 1nterface, and on-line graphic display programs show the data as they are being accumulated. The data acquisition aystem has been described by Garner. ${ }^{29}$ No event by event logging of data is done; the raw data consist of these histograms. An overall time resolution of approximately 2 ns is achleved.

To obtain a histogram which reflects the time distribution accurately, certain "bad" events must be suppressed. In order that a given position can be associated with the muon from which it was emitted, we require that only one muon be present in the sample at one time. A pileup gate (Model GP 100/N, EG\&G 0rtec), known as the "data gate", Is used to allow a clock start only when a $\mu^{+}$arrives at least a time $T$ after the last previous $\mu^{+}$, where $T$ is chosen to be several $\mu^{+}$lifetimes. The pileup gate 1 s also used to reject events where a second $\mu^{+}$arrives within time $T$ of a $\mu^{+}$which started the clock. Events are also rejected if more than one positron is seen during the period $T$ after the arrival of the $\mu^{+}$. Timing diagrams for typical "good" and "bad" events are shown 1n Flg. 6.

The two-muon rejection effects a limitation on the ultimate rate at which one accepts $\mu^{+}$. If the arrival times of the $\mu^{+}$are governed by Poisson statistics, then the "good" event rate $R_{g}$ is related to the incident muon rate $\mathrm{R}$ and the gate length $\mathrm{T}$ by ${ }^{29^{\circ}}$

$$
R_{8}=R \cdot \exp (-2 T R) \text {. }
$$

$R_{g}$ exhibits a maximum at $R=(2 T)^{-1}$, so with a typical gate width of 10 13 the maximum usable incident muon rate 13 about $50 \mathrm{kHz}$, readily achleved at IRIUMF. At this rate we get typically $8,000-10,000 \mathrm{e}^{+} / \mathrm{s}$, 
depending somewhat on the target geometry and placement.

In using Polsson statistics, we have Implicitly assumed that the cyclotron beam has no time structure. At TRIUMF under normal operatIng conditions the time structure occurs with a pertod of $43 \mathrm{~ns}$, which is sufficiently smaller than the $\mu^{+}$Iffetime that the Poisson assumption is valid. If the beam had a duty factor corresponding to a time structure of period much greater than a muon lifetime, then the effective rate would be decreased by that factor.

In practice, $T$ is usually selected to be at least $8 \mu$, at which time only $2 \frac{1}{2} z$ of the muons will remain. In principle, T could be made much shorter if one could make corrections for the incomplete rejection of "bad" evenics, or If one wants only to compare different samples. In practice, this can be dangerous, as the resulting distortions to the spectra are dependent on the Instantaneous (up to - I MHz) $\mu^{+}$arrival rate, which may fluctuate with time.

All events which appear "good" at the time of the clock stop pulse activate "e Gate", which causes a CAMAC service routine to be requested at the end of the data gate. If subsequent second muons or electrons arrive, a reject bit will be set so the event will be rejected by the software. A dead-time gate is activated on a "good $e^{\prime \prime}$ and Inhibits sensitive portions of the logic until cleared at the end of the CAMAC service routine. It is very important, however, that the pileup gate run continuously 1 s order to keep track of $\mu^{+}$ arriving during the "computer busy" condition. 
The rejection of second muons is the primary feacure of the data acquisition aystem, especially since on the $49-$ W3 beam Iine there are no beam electrons so one in principle rarely gets a second $e^{+}$without a corresponding second muon if $T$ is long enough. But if the beam counter is not $100 \%$ efficient for $\mu^{+}$, then some muons are able to "leak" in and cause distortions in the spectra. The requirement that $D$ be highly efficient for $\mu^{+}$is somewhat contradictory to the requirement that its discriminator be set $h 1 g h$ enough to reject beam electrons. If a beam positron can fire D to give a start pulse, then a subsequent beam positron can scatter off this sample to give a "stop". Due to the time structure of the beam, this will appear as a 23 MHz nolse signal in the spectrum.

An additional type of beam noise occurs when a beam positron fires an "e" colncidence after a muon caused a start. Th1s will cause an exponential background to the data which actually appears flat if the beam electron rate 1s low. The carbon moderator suppresses these to a large extent, and in general it is better to leave the D discriminator set a bit low. It is much more harmful tc get a few second muons than a few second electrons.

The problems with beam electrons are completely eliminated by the use of the electron-free M9-W3 beam, and the D discriminator can be set with a low enough threshold so as to be essentially 100\% effic1ent.

The rejection of "bad" events is tested for by examining least squares fits to the observed spectra in transverse fleld of standard 
targets, usually high purity aluminuol. In the case of the "e-start, e-stop" events, examination of the Fourler transform for $23 \mathrm{MHz}$ noise is used. On-line curve fitting and fast Fourler transform programs are available on the data acquisition computer so that these tests may be done as necsssary during an experiment. On M9-W3, essentially perfect (ch1-squared between 0.9 and 1.1 ) fits to high-statistics ( $3 \times 10^{6}$ eventg/h1stogram) data with backgrounds of $\leqslant 17$ are obtained and no $23 \mathrm{MHz}$ is visible, indicating very adequate "bad" event reject1on.

\section{Cryogenics}

c.. l. $\quad \underline{2} \underline{\mathrm{K}}$ toㅇ $300 \underline{\mathrm{K}}$

Low-temperature experiments are conducted with a ${ }^{4}$ He gas-flow cryostat (Model 10DT Super-VariTemp, Janis Research Co., Stonehan, Ma. 02180) with an optical tail extending down into the beam. Samples are Inserted through the top with a removable positioner. Tue outer tall, which serves as a vacuum shield, is removed to eliminate its window from the total thickness the $\mu^{+}$must traverse. The cryostat Insulating vacuum is contiguous to that surrounding the counters.

The $\mu^{+}$pass a $0.0003^{\prime \prime}$ aluminized mylar window in the $77 \mathrm{~K}$ radiation shield and a $0.005^{\prime \prime}$ mylar pressure window separating the sample space frum the vacuum. The targets are mounted as close to this window as possible, because ${ }^{4} \mathrm{He}$ gas at $4.2 \mathrm{~K}$ has sufficient density $\left(0.017 \mathrm{~g} \mathrm{~cm}^{-3}\right)$ to cause appreclable loss of $\mathrm{\mu}^{+}$range over only a few cm. Accumulation of liquid ${ }^{4}$ He up to the window level must be 
avolded entirely, as $1 \mathrm{~mm}$ of it can completely stop the beam.

The temperature is controlled by a heater on the vaporizer assembly and by adjusting witl: a needle valve the flow of 4 He into the vapor1zer. Adequate temperature stability ( $\pm 0.05 \mathrm{~K}$ at $5 \mathrm{~K}$ ) for many experiments is obtained by running the heater at constant current and taking care that the pressure in th. ${ }^{4}$ He reservoir, which drives the 11quid ${ }^{4} \mathrm{He}$ flow, remains constant. For more precise temperature stability, feedback from a thermometer on the sample can be used.

To obtain temperatures below $4.2 \mathrm{p}$, a mechanical rotary pump was used to reduce the pressure in the sample area. Care must be taken so that liquid ${ }^{4} \mathrm{He}$ is not drawn up around the sample when dotrg, this. Temperatures down to about $2 \mathrm{~K}$ can be achleved in this fashion.

\section{C.․․ $\quad$ Below $\underline{2} \underline{\mathrm{K}}$}

For temperatures below $2 \mathrm{~K}, \mathrm{a}^{3} \mathrm{He}$ single-shot evaporation refrigerator ( $\mathrm{FH}_{\mathrm{g}}$. 7) was fabricated. It consists of a copper cell containing several $\mathrm{cm}^{3}$ of ${ }^{3}$ He surrounded by an insulating vacuum space and upon which up to four samples are mounted with GE 7031 varnish. By pumping on the ${ }^{3}$ Ie with a closed-cycle mechanical pumping station temperatures down to $0.5 \mathrm{~K}$ can be reached. The celi holds enough ${ }^{3}$ He to maintaln $0.5 \mathrm{~K}$ for $>6 \mathrm{~b}$.

The ${ }^{3}$ He refrigerator assembly was made as an insert for the Janis 4 ife cryostat and is easily rotated to present the different samples to the beau. The vacuur/exchange-gas space around the reser- 
voir is maintained by a surrounding can, provided with four 0.005" aluminized mylar windows for the beam. The Janis cryostat is used to reach $2 \mathrm{~K}$ with $-100-200 \mu^{3} \mathrm{He}$ exchange gas present, ${ }^{3} \mathrm{He}$ is condensed 1n the cell, and the exchange gas is pumped out. The system 1s then ready for the ${ }^{3}$ He cell to be pumped.

A carbon composition resistor was mounted or the bottom of the exchange gas can to sense 1iquid ${ }^{4}$ He build-up in the tail of the Janis cryostat. Sample temperatures were measured with a calibrated Ge res1stor (Mode1 GR-200A-100, Lakeshore Cryotronics, Westerville, Ohio 43081) glued with GE varnish to the ${ }^{3}$ He cell. Temperature was adjusted with metering valves on the ${ }^{3}$ He pump inlet.

With this refrigerator in place, the total mass in the $\mu^{+}$beam upstream from the sample is approximately $90 \mathrm{mg} \mathrm{cm}-2$, Including the two $0.001 "$ Kapton w1 ndows in the d.c. separator on M9-W3.

\section{Data Analysis}

The observed spectrum for the $1^{\prime}$ th telescepe $1 s^{1}$

$$
N_{i}(t)=N_{O 1} \cdot\left[e^{-t / \tau}\left[1+A_{1} \vec{p}_{\mu}(t) \cdot \widehat{n}_{i}\right]+B_{i}\right]
$$

where $N_{o 1}$ is an overall normalization, $A_{1}$ is the instrumental asymmetry of the telescope (the asymmetry that is seen for $100 \% \mu^{+}$polarization), $\vec{p}_{\mu}$ is the $\mu^{+}$ensemble polarization, $\hat{\mathbf{n}}_{1}$ are the telescope Afrections, B is a constant background term, and $t=2.197 \mu$ s is the $\mu^{+}$IIfetime. The data are analyzed $1 n$ the simplest case by independent least-squares fits to 1ndividual telescopes, allowing $N_{0}, A, B$, and any parameters in $\vec{p}_{\mu}(t)$ to float. This is valuable in order to 
check for agreement among the telescopes, hut more properly one should calculate a chI-squared as a sum on all the telescopes since the parameters intering into $\vec{p}_{\mu}$ are common to all telescopes. In prartice, with 4 telescopes this becomes difficult as too many parameters result for the available computer programs to vary simultaneously, so we Instead fit two opposing telescopes at a time when using this method. $t$ may also be floated for an empirical check of bad event refection since two-muon events lead to an apparent $\mu^{+} 11$ fetime shorter than $2.2 \mu s$. The MIrUIT ${ }^{30}$ minimization program was used to obtain least-squares fits to the data and to generate statistical errors.

In a transverse-field experiment the spectra become

$$
N_{1}(t)=N_{o 1}\left(e^{-t / \tau}\left[1+A_{1} G_{x}(t) \cos \left(u t-\phi_{1}\right)\right]+B_{1}\right\}
$$

where $\phi_{1}$ is the angle of the $1^{\prime}$ th telescope with respect to the beam direction and we have introduced the transverse relaxation function $G_{X}(t) \cdot I_{-\sigma}$. Ba shows a typical example of a trangverse-field $\mu^{+} S R$ spectrum, and $F 1 g$. $8 b$ shows the asymmetry $A G_{x}(t) \cos (w t-\phi)$ obtained from the raw data by dividing by $N_{0} e^{-t / \tau}$ after subtracting $N_{0} B$. The envelope of the oscillatious in FIg. 8b is the Interesting 1nformation provided by the experiment.

In the longltudinal geometry, only two telescopes (B,F) give any Information, and the spectra are

$$
\mathrm{N}_{E}=\mathrm{N}_{O F}\left\{e^{-t / \tau}\left[1-A_{F} G_{2}(t)\right]+B_{E}\right\}
$$




$$
N_{B}=N_{O B}\left\{e^{-t / \tau}\left[1+A_{B} G_{2}(t)\right]+B_{B}\right\} \text {. }
$$

In this case, the damping of the polarization is described by the longltudinal relaxation function $G_{z}(t)$, which is in general not the same as $G_{x}(t)$.

Becauge of the very shcrt range of the surface $\mu^{+}$, few $\mu^{+}$stop outside of the sample. For this reason, it is unnecessary to perform background subtractions if the sample is large enough to fill the beam spot. Subtractions of this sort are performed in the experiments of Ref. 23 and contribute to their difficulty in determining the shape of $G_{x}(t)$. Experiments on high purity silicon at $15 \mathrm{~K}$ wth this spectrometer on M9-W3 have shown diamagnettc asymmetry less tiran 0.005 , which sets an upper 11mit of -27 for the number of $\mu^{+}$stopping in the diamagnetic material around the sample. 
THE DIPOLAR RELAXATION OF THE $\mu^{+}$

We now tura to a more detailed examination of $\mathrm{p}^{+}$spin relaxation phenomena than that presented in the Introduction. As mentioned there, relaxation of the $\mu^{+}$polarization can be caused by local magnetic Elelds from the randomly orlented nuclear dipoles in the material. It is the purpose of this chapter to discuss the nuclear dipolar spin relaxation of the $\mu^{+}$with an cmphasis on the relationship of the transverse-field and zero-field methods. Huch of the material in this chapter has been discussed in publications of the Dniversity of Tokyo group, $4,31,32$ who have performed semiclassical calculations of moments and relaxation functions. Here we use a quantum mechanical approach where possible, since this proves to be necessary in order to understand the effect of the nuclear electric quadrupole interaction.

Since frequency units are the most natural in this work, we will use units such that $\pi=1$ chroughout th1s chapter.

The general quantum mechanical problem is to find the elgenvalues and eigenstates of the Hamiltonian for the coupled spin system of the $\mu^{+}$and $N$ Idencical nuclei in the applied field $\overrightarrow{\mathrm{B}}_{\mathrm{o}}$ :

$$
\boldsymbol{H}=\mathbf{Z}_{\mathbf{Z}_{\boldsymbol{\mu}}}+\mathbf{H}_{\mathbf{Z I}}+\mathbf{H}_{\boldsymbol{\mu} I}+\mathbf{H}_{I I}
$$

where ${ }^{Z}{ }_{Z I}$ and $H_{Z I}$ are the muon and nuclear Zeeman energies, and $H_{\mu I}$ and $\mathrm{H}_{\mathrm{II}}$ are the muon-nucleus and nucleus-nucleus Interactions. He 
can break $H$ Into an unperturbed Zeeman Hamiltonian ${ }^{33}$

$$
\mathrm{H}_{\mathrm{o}}=-\left(\mathrm{Y}_{\mathrm{H}_{\mathrm{z}}}+\mathrm{Y}_{\mathrm{I}} \sum_{1} \mathrm{I}_{\mathrm{z}}^{\mathrm{L}}\right) \mathrm{H}_{\mathrm{O}}
$$

and a perturbing dipolar Hamiltowian

$$
\begin{aligned}
H_{d} & =y_{\mu} y_{I} \underset{1}{\sum} \frac{1}{I_{1}^{3}}\left[\vec{s} \cdot \vec{I}^{1}-3\left(\vec{s} \cdot r_{i}\right)\left(\vec{I}^{1} \cdot \vec{r}_{1}\right)\right] \\
& +\gamma_{I}^{2} \underset{j<k}{\sum} \frac{1}{r_{j k}^{3}}\left[\vec{I} j \cdot \vec{I} k-3\left(\vec{I}^{j} \cdot q_{j k}\right)\left(\vec{I}^{k} \cdot q_{j k}\right)\right] .
\end{aligned}
$$

Here $\vec{s}, \vec{I}^{j}$ are the spin operatorg of the muon and tise $j$ th nucleus, $Y_{\mu}, Y_{I}$ are their respective gyromagnetic ratios, $\vec{r}_{1}$ is the position

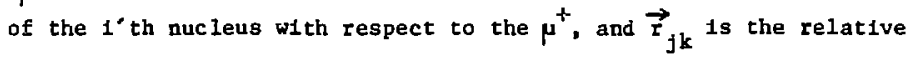
position of the $j^{\prime}$ th and $k^{\prime}$ th nuclei.

Hote the relative magnitudes of the two terms of $\mathrm{H}_{\mathrm{d}}$. The term ${ }^{H_{I I}}$ is much smaller than ${ }_{\mu I}$ since $y_{\mu} \gg Y_{I}$ (for copper, $y_{\mu} / Y_{I}=11.7$ ). The effect of this term is to cause nuclear spin flips at a rate $-0.01 \mathrm{\mu s}^{-1}$, which $1 \mathrm{~s}$ negliglble for our purposes, and we drop $\mathrm{H}_{\mathrm{II}}$ in al1 subsequent work. In the particular case of copper, it has been shown $^{34}$ experimentally that the nuclear spin relaxation rate is small $\left(\sim 0.01 \mathrm{\mu s}^{-1} \mathrm{in}^{63} \mathrm{Cu}\right)$ and cemperature independent.

\section{A. The Static Relaxation}

We assume in this section that the $\mu^{+}$is fixed in position, so that all $\vec{r}_{1}$ in Eq. III. 1 are constant in time. The density matrix formallsm is used to find the ensemble average of the expectation value of the $\mu^{+} \operatorname{spin}$ operator $\overrightarrow{s_{+}}$. This is given in the Heisenberg representation by 


$$
\begin{aligned}
\langle\vec{s}(t)\rangle & =\operatorname{tr} \overrightarrow{p s}(t) \\
& =t r p e^{1 \text { Ht }} \vec{s} e^{-1 H t},
\end{aligned}
$$

where $p$ is the (time Independent) density operator of the $\mu^{+}$-nuclei system. We take $\overrightarrow{\mathbf{s}}$ written with no argument to be the Pauli spin- $1 / 2$ operator $\vec{s}=1 / 2 \vec{\sigma}$.

Since we shall always have $k T \gg y_{I}{ }_{0}$, we as sume the nuclel are unpolarized. With the density matrix for a $\mu^{+}$with inttial polarization $\vec{p}, p_{\mu}=1 / 2+\vec{p} \cdot \vec{s}$, this yields for the total density natrix for the syatem,

$$
P=\frac{1}{(2 I+1)^{N}}(1 / 2+\vec{p} \cdot \vec{s})
$$

The desired quantity becomes

$$
\langle\vec{s}(t)\rangle=\frac{1}{(2 I+1)^{N}} \operatorname{tr}[(\vec{p} \cdot \vec{s}) \vec{s}(t)\rfloor,
$$

where we have dropped the term $t r \vec{s}(t)=0$.

\section{A-1. Transverse-field}

We have $\mathrm{H}_{0} \| z, \overrightarrow{\mathrm{p}}(0)=\mathrm{R}$ and we seek $\left\langle s_{f}(t)\right\rangle$. Eq. III. 2 reduces in this case to 35

$$
\begin{aligned}
\left\langle s_{+}(t)\right\rangle & =\frac{1}{2(2 I+1)^{N}} \operatorname{tr}\left[s_{-} s_{+}(t)\right] . \\
& \propto \exp \left(-i{w_{0}}_{0} t\right) \operatorname{tr}\left[s_{-} s_{+}(t)\right]
\end{aligned}
$$

where $s_{+}(t)$ is the interaction representation operator

$$
g_{+}(t)=\exp \left(-1 \mathrm{H}_{0} t\right) \mathrm{s}_{+}(\tau) \exp \left(1 \mathrm{H}_{0} t\right),
$$

and $w_{0}=y_{\mu} H_{0}$ is the center frequency of the 11 ne. From this we see 
that the envelope of the oscillations is given by the relaxation function

$$
G_{x}(t)=\frac{\operatorname{tr}\left[s_{-} s_{t}(t)\right]}{t r\left[s_{-} s_{+}\right]} .
$$

The remalning plece of $\mathrm{H}_{\mathrm{d}}$, that $1 \mathrm{~s}, \mathrm{H}_{\mu I}$, can now be written in terms of the spherical angular momentum components, which will help us to see the effects of the varfaus terms. This is a standard procedure in NMR ${ }^{36,37}$ and we will not give all the algebra. The result Is

$$
\begin{aligned}
& \#_{d}=\gamma_{\mu} \gamma_{I} \sum \frac{1}{r_{1}^{3}}\left[\left(1-3 \cos ^{2} \theta_{1}\right) s_{z} I_{z}^{1}\right. \\
& \left.+\left(\text { terms in } s_{ \pm} I_{\mp}, s_{z} I_{ \pm}, s_{ \pm} I_{z}, s_{ \pm} I_{ \pm}\right)\right] \text {, }
\end{aligned}
$$

where $\theta_{1}$ is the polar angle of $\vec{r}_{1}$.

From this it is easy to see that only the term in $s_{z} I_{z}$ (known as the secular term), which commutes with $\mathrm{H}_{0}$, can cause a first order tnergy shift of any muon Zeeman level. The effect of the non-secuiar teris is to cause slight admixtures of states of differing energy;

$$
\begin{aligned}
\left|m_{\mu}, m_{I}\right\rangle \rightarrow\left|m_{\mu}, m_{I}\right\rangle+\lambda \cdot & {\left[\left|m_{\mu} \pm 1, m_{I}\right\rangle+\left|m_{\mu}, m_{I} \pm 1\right\rangle\right.} \\
& \left.+\left|m_{\mu} \pm 1, m_{I} \mp l\right\rangle+\left|m_{\mu} \pm 1, m_{I} \pm 1\right\rangle\right],
\end{aligned}
$$

where $\lambda$ is symbolic of a small quantity which decreases rapidly with the unperturbed energy differences. The result of this is to allow weak transitions at frequencles far from the line of 1nterest, the closest being at $u_{0} \pm y_{I} H_{0^{*}}$ Only if these satellite 11nes approach 
within approximately a line width $(\sigma)$ of the main line can they appear as a broadenlag; otherwlse they appear as separate lines. For $w_{I}=y_{I} H_{0}>\sigma$ these terms do not contribute to the width of the maIn IIne at $w_{0}$

This language is that of NMR, where one stually observes in the frequency domain. In the $\mu^{+} \mathrm{SR}$ experiment one observes in the time doma1n, but one measures the relaxation by curve fitting as if only a single frequency were present. In fact, the satellite resonances are far too weak to be observed by $\mu^{+} \mathrm{SR}$. Thus the same reasoning applies In our case, and henceforth we proceed using the "truncated" dipolar Hamiltonlan:

$$
H_{d}^{\prime}=\gamma_{\mu} y_{I} \sum \frac{1}{r_{1}^{3}}\left(1-3 \cos ^{2} \theta_{1}\right) s_{z} I_{z}^{1}
$$

Since $H_{d}$ is just proportional to $g_{z}$, it is now easy to obtain an exact solution for $G_{x}(t)$. If we define $b_{1}=r_{1}^{-3}\left(1-3 \cos ^{2} \theta_{1}\right)$, the result $1 \mathrm{~s}$

$$
G_{x}(t)=\prod_{1=1}^{N} \frac{1}{2 I+1} \sum_{m_{1}=-I}^{I} \exp \left[1 y_{\mu} y_{I} b_{1} w_{1} t\right] \text {. }
$$

By expanding the logarithm of $G_{x}$ in powers of $\beta_{1}=1 / 2 y_{\mu} y_{I} b_{1} t$, we obtain

$$
G_{x}(t) \Rightarrow \exp \left[-1 / 2 \sigma_{v v^{2}}^{2}\right]
$$

where

$$
\sigma_{V V}^{2}=M_{2}^{t}=\frac{1}{3} y_{\mu}^{2} y_{I}^{2} I(I+1) \underset{1}{\sum} \frac{1}{r_{1}^{6}}\left(3 \cos ^{2} \theta_{1}-1\right)^{2}
$$


is the Van Vleck ${ }^{14}$ value for the second moment. Note that this is also the second moment of the exact form as can be verified by differentiating Eq. III. 3.

It should be noted that it has become conventional in experimental transverse-field $\mu^{+}$SR to define $G_{x}(t)=\exp \left(-\sigma^{2} t^{2}\right)$. The parameters are related by $\sigma=\sigma_{\mathrm{VV}} / \sqrt{2}$.

\section{A. 2. Zero-field}

In this case we use the initial ${ }^{+}$spin direction as the $z$ axis; we have $\vec{p}(0)=\hat{z}$ and we seek

$$
G_{z}(t)=\frac{\operatorname{tr}\left[s_{z} s_{z}(t)\right]}{\operatorname{tr}\left[s_{z}^{2}\right]} .
$$

In the zero-field case we can not justify dropping the nonsecular terms because there is no strong applied field to cause rapld precession of the nuclel. As the field is reduced to zero, the satellite lines which these terms create will grow and approach the ma1n line and broaden 1t. Unfortunately, no simple solution for the relaxation function is possible in this case since the different components of $H_{d}$ do not commute with one another. We can, however, use the method of moments in order to evaluate the zero-field second moment $M_{2}^{2 f}$ using the full dipolar Hamiltonian $\mathrm{F}_{\mathrm{d}}$. This is done in an appendix; the result is

$$
M_{2}^{z f}=\frac{1}{3} y_{\mu}^{2} y_{I}^{2} I(I+1) \underset{1}{\Sigma} \frac{1}{r_{1}^{6}}\left(5-3 \cos ^{2} \theta_{i}\right) .
$$

A classical calculation of $y_{\mu}^{2}\left(\mathrm{H}_{x}^{2}+\mathrm{H}_{y}^{2}\right)$ keeping all components of $\vec{\mu}_{I}$ 
gives this same result. 4

Note that for a $\mu^{+}$site with cubic symmetry, the sum of $\cos ^{2} \theta_{1}$ over any shell of netghbors giveg fust the spherlcal average, $\left(\cos ^{2} \theta\right)=1 / 3$. In this case the zero-field width, in contrast to the transverse-fleld width, is 1sotroplc. This will be the case, for Instance, for the octahedral site In an fcc lattice.

The zero-field relaxation function can also be calculated ilrectly from the Gaussian assumption, although the line shape 1s not Bimply an Image of the local field distribution as in the transverse-field case. For a $\mu^{+}$in a local field $\overrightarrow{\mathrm{H}}=\left(\mathrm{H}_{x}, \mathrm{H}_{\mathrm{y}}, \mathrm{H}_{z}\right)$, the time dependence $i f$ the $a$ component of the spin expectation value is

$$
s_{z}(\vec{H}, t)=\cos ^{2} \theta+\sin ^{2} \theta \cos \left(\gamma_{\mu}|\vec{H}| t\right) \text {, }
$$

where $\theta$ is the polar sable of $\vec{B}$. If the local field is distributed according to $P(\vec{H})$, the observed relaxation function is $^{4}$

$$
G_{z}(t)=\int P(\vec{H}) s_{z}(\vec{H}, t) d^{3} \vec{H}
$$

(A similar approach may be used in an applied field.) If the local fleld distribution is isotroplc, $P(\vec{B}) d^{3} \vec{H}=H^{2} P(H) d H d Q$, we have

$$
G_{z}(t)=\frac{1}{3}+\frac{2}{3} \int H^{2} P(H) \cos \left(Y_{\mu} H t\right) d H .
$$

For $P(H)$ a Gaussian with width $y_{\mu}^{2}\left(H_{1}^{2}\right)=\Delta^{2}$, we arrive at ${ }^{4,38}$

$$
G_{z}(t)=\frac{1}{3}+\frac{2}{3}\left(1-\Delta^{2} t^{2}\right) \exp \left(-1 / 2 \Delta^{2} t^{2}\right) \text {. }
$$

This function shows some interesting behavior: it initially decays as a Gaussian, $\exp \left(-\Delta^{2} t^{2}\right)$, and has a minimum at $c=\sqrt{3} \Delta^{-1}$ followed by a recovery of the polarization to an asymptotic value of 
$1 / 3$ at time $t \gg \Delta^{-1}$ (see the curve labeled $V=0$ in F1g. 9a). The recovery to $1 / 3 \mathrm{is}$ of special interest, as that particular feature is Independent of the actual distribution In magnitude of the local field, as can be seen directly from Eq. III.7. Such behavior depends only on the local field being 1sotropic and static.

The behavior of the minimum at $t=\sqrt{3} \Delta^{-1}$ does depend on the Gaussian assumption. If one assumes a Lorentzian distribution of local fields, as in a dilute assembly of dipoles with random positions, ${ }^{39}$ one finds a zero-field relaxation which exhibits only a very shallow minimum. 38,40

The recovery of the polarization to $1 / 3$ is very sensitive to changes in the local field duriag the muon lifetime, as this wil cause the $\mu^{+}$spin to lose its memory of its initial direction. This will be made more quantitative in section $B$.

\section{A.}

To compare these formulae for $\mathrm{M}_{2}$, we take a polycrystalline average to eliminate the angular dependence. With $\Delta_{x}^{2}=y_{\mu}^{2}\left(H_{z}^{2}\right)$ we find for the transverse-field case,

$$
\overrightarrow{M_{2}}=\Delta_{x}^{2}=\frac{4}{15} y_{\mu}^{2} y_{I}^{2} I(I+1) \underset{1}{\sum} \frac{1}{6}
$$

For the zero-field case, with $\Delta_{z}^{2}=y_{\mu}^{2}\left(\mathrm{H}_{x}^{2}\right)$, we get

$$
\vec{M}_{2}^{2 f}=2 \Delta_{z}^{2}=\frac{4}{3} y_{\mu}^{2} y_{I}^{2} I(I+1) \underset{1}{\sum} \frac{1}{r_{1}^{6}}
$$


We see that the zero-field second moment is a factor of 5 larger than the transverse-field value; the high field reiaxation function initially decays only (5) ${ }^{1 / 2}$ as rapidly as the zero-field function. Note that this is a combination of 2 effects. As the field is lowered to a value such that $w_{I} \sim \sigma$ the cerms in $s_{z} I_{t}$, corresponding to the $x$ and $y$ components of $\vec{\mu}_{I}$, can now bradden the main line, which gives us the ratio of mean-square dipolar field components $\Delta \Delta_{z}^{2} / \Delta_{x}^{2}=5 / 2$. The nuclear dipoles are not precessing rapidly enough to average out the $1 \mathrm{r} x$ and $y$ components. As the field is lowered further so that $u_{0}-\sigma$, we recover the terms containing $s_{ \pm}$. The $x$ and $y$ components of the local field are now simflar in magnitude to the $z$ component and hence equaliy effective in relaxing the spin.

\section{ㅌ. Dynamic Effects}

The results presented in the preceding section are based on the assumption that random field components are elther static or varying rapldly enough to be averaged out to zero. This section will be concerned with the case where hopping of the $\mu^{+}$causes fluctuations in the local fleld to occur during its 11fetime.

Formally, motion of the $\mu^{+}$is obtained by adding an additional term to the Hamiltonian representing the $\mu^{+}$kinetic energy and Its Interaction with the lattice. The effect of this term can then be calculated by perturbation theory. 41 This leads to correlation functlons which would be extremely difficult to evaluate. An approach more amenable to calculation is to let $\mathrm{H}_{d^{\prime}} \rightarrow \mathrm{H}_{\mathrm{d}}{ }^{\prime}(\mathrm{t})$, a random function of time, and make simple assumptions about 1ts behavior. 
In the transverse-field case, the relaxation function, equation III. 3, can be thought of as the average $\langle\exp (-10 t))$, where the average is taken over the vartous nuclear spin configurations and $\Omega=-\sum_{i} \gamma_{\mu} y_{I} b_{1}{ }_{1}$ fs the shift in the $\mu^{+}$Larmor frequency generated by the nuclear spin configuration $\left\{m_{i}\right\}$. If we allow $H_{d}^{*}$ to be time dependent, the time evolution operator becomes $\exp \left[-i \int_{0}^{t} \mathrm{H}_{d}^{\prime}\left(t^{\prime}\right) \mathrm{d} \mathrm{t}^{\prime}\right]$ and in terms of $\ell$ we have

$$
G_{x}(t)=\left\langle\exp \left[-i \int_{0}^{t} \Omega\left(t^{\circ}\right) d t^{-}\right]\right\rangle \text {. }
$$

Making the assumptions that $Q$ is a stationary Gaussian process with $\langle\Omega(t) \Omega(t-\tau)\rangle=\sigma^{2} \exp [-\nu|\tau|]$, it is found that 12,13

$$
G_{x}(t)=\exp \left[-\sigma^{2} v^{-2}\left(e^{-V t}-1+\nu t\right)\right] \text {. }
$$

In the 11mit $\Downarrow \rightarrow 0$ we recover the previous result

$$
\lim _{V \rightarrow 0} G_{x}(t)=\exp \left[-1 / 2 \sigma^{2} t^{2}\right] \text {, }
$$

whereas in the Iimit of rapid fluctuations we obtain

$$
\lim _{v \rightarrow \infty} G_{x}(t)=\exp \left[-\sigma^{2} v^{-1} t\right] \text {. }
$$

This exhibits the phenomenon of motional narrowing. As the $\mu^{+}$begins diffusing more rapidly, the line becomes narrower and its shape changes from Gaussian to Lorentzian. In the limit of extremely rapid modulation, no relaxation occurs.

For hopping rates $\nu \ll u_{I}, \sigma^{2}$ will retain 1 ts static value of $\sigma_{\mathrm{VV}}^{2}$. For more rapid hopping, the rapldly fluctuating fields can 
facilitate recovery of the non-secular terw3, 42 with a concomitant increase in $\sigma^{2}$. The application of this to $\mu^{+}$spin relaxation has been discussed by Hayano, et al. 4

The application of the stochastic model to low- and zero-field relaxation was first considered by Kubo and Toyabe ${ }^{38}$ in 1967. With the assumption of a Gaussian process for $\vec{H}(t)$, they derived $G_{z}\left(D, \Delta_{z}, t\right)$, recovering equation III.8 In the static limit. The sensitivity of the polarization for $t \geq \sqrt{3} \Delta_{z}^{-1}$ to the hopping rate $U$ was apparent from their treatment, but the problem was seen as academic due to the Inability to measure the zero-field spin relaxation.

A further treatment was given recently by Hayano et al. 4,43 Instead of treating $\vec{H}(t)$ as a Gaussian process with an exponential correlation function, they assumed a strong colliston model where the field after a hop is uncorrelated with that before the hup. This allows an exact solution for $G_{z}(t)$ to te obtained which is more suitable for numerical computation. The strong collision model gives essentially the same results as the kubo-Toyabe model, although the latter glves a slightly stronger decay in the slow hopping $(V / \Delta<1)$ regime.

Curves of $G_{z}(t)$ and $G_{x}(t)$ with $\Delta_{z}^{2}=5 / 2 \Delta_{x}^{2}$ are shown in Fy. 9. In order to see the relationship of the widths, the time axis in both cases is $\Delta_{z} t$. Because of the larger second moment, $G_{z}(t)$ decays more rapidly than $G_{x}(t)$. Also apparent is that a similar motional narrowing occurs in both for $W / \Delta \geq i$. More important for many purposes, though, is the behavior of $G_{2}(t)$ for $t \geq \sqrt{3} \Delta_{2}^{-1}$. At 
those Iong times, the recovery of the polarization to $1 / 3$ is very sensitive to hoppling with $W / \Delta<1$. Thus, in addition to motional narrowing, $G_{2}(t)$ exhibits "motional decay of the asymptotic polarization", or "motional bLoadenting of the zero-frequency transition", which is far more sensitive to slow fluctuations of the local field.

The strong collision model has also been shown to be sultable for computation by a Honte Carlo treatment of the random fleld and the hopping process. ${ }^{44-46}$ Kubo 40 has given a general treatment of the stochastic model from which these different models can be derived by making the appropriate assumptions about the process $\overrightarrow{\mathrm{H}}(t)$.

\section{The Quadrupole Interact1on}

In the preceding sections of this chapter, we have been concerned only with the zeeman levels in the applied field $\vec{H}_{0}$ and the magnetic dipole interaction between the $\mu^{+}$and the nucles. However, the naturally occurring 1sotopes of copper, ${ }^{63} \mathrm{Cu}$ and ${ }^{65} \mathrm{Cu}$, both have $\operatorname{spin} 3 / 2$ and electric quadrupole moments $Q=-0.15 \times 10^{-24} \mathrm{~cm}^{2}$. An electric fleld gradient (EFG) caused by the lattice expansion about ine $\mu^{+}$exists at the Cu nucle1, and the interaction of the EFG with their quadrupole moments has a strong effect on the dipolar relaxation.

The total Hamiltonlan is now (assuming an axially symmetric quadeupole interaction)

$$
\mathrm{H}=\mathbf{R}_{\mathrm{Zu}}+\mathbf{H}_{\mathrm{ZI}}+\mathbf{B}_{\mathrm{Q}}+\mathrm{B}_{\mathrm{d}},
$$

where 


$$
\mathrm{H}_{\mathrm{Q}}=\mathrm{w}_{\mathrm{Q}} \cdot\left[3 I_{\mathrm{z}}^{2}-I(I+1)\right] \text {. }
$$

The $z$ axis is here taken, along the direction of the EFG, which is different for each nucleus. Note that $H_{Q} 1$ tself contalns no $\mu^{+}$ operators. Its effect on the $\mu^{+}$spin 1 s to change the way in which the nuclear dipoles precess, therefore changing the truncation to be performed on $\mathbb{H}_{d}$.

\section{Transverse-tield}

In transverse field, the Importance of the quadrupole Interaction can be parametrized by the ratio of magnetic to electric energles, $w_{I} / w_{Q}$ (n.b.--for a spin-3/2 nucleus, the quadrupole splitting is actually $\left.6 w_{Q}\right)$. This has been treated theoretically by Hartmann. 11 He has shown that if the magnetic field is sufficiently strong $\left(w_{I} / w_{Q}>l\right)$, the dipolar width $\sigma_{V V}^{2}$ (equation III.5) is seen, whereas in lower fields the relaxation is changed from this value. The reason is that the $z$ axis (magnetic field direction) is no longer a unique quantization axis since nuclel now tend to precess about the directions of the vectors $\vec{r}_{1}$ connecting the $\mu^{+}$with each nucleus. In low fields, this precession can cause averaging of non-secular terms just as precesston about $\overrightarrow{\mathrm{H}}_{\mathrm{o}}$ can.

This is treated formally in a similar fashion as the truncation of the dipole hamiltonian in the high fleld case, section III.A, except the unpercurbed Hamiltonian of the nuclei is now $\mathrm{H}_{\mathrm{I}}^{\mathrm{T}}=\mathrm{H}_{\mathrm{ZI}}+\mathrm{H}_{\mathrm{Q}}$. The energles and elgenstates of this are generally not simple, and one must numerlcally diagonalize ${ }^{\mathrm{T}}$ and truncate the nuclear spin operators appearing in $\mathrm{H}_{\mathrm{d}}$ to remove the matrix elements 
connecting different unperturbed energles. The $\mu^{+}$spln operators are treated the same as before since $H_{Q}$ thas no direct effect on the $\mu^{+}$. In the experiments of Caman1, et al. $7,9,10$ this theory was applied to measurements of the orfentation and field dependence of the transverse-field relaxation rate. The data with theoretical curves are shown In Fig. 10. There are two princlpal effects that are noted.

(1) The high-field relaxation rates are given by the Van Vleck values with the nearest neighbor distances Increased by $4.9 \%$. From the high-fieid orlentational dependence, it ig concluded that the $\mathrm{\mu}^{+}$resides in an octahedral interstitial site.

(2) The orientational dependence of $\sigma$ is weakened by the quadrupole Interaction at low fields. From the field dependence of $\sigma$, it Is concluded that $w_{Q}=1.1 \pm 0.1 \mu^{-1}$.

\section{$\underline{\text { Zero-field }}$}

In zero fleld, Hartmann's calculations do not apply, but in this case the total unperturbed Hamiltonian is easily diagonalized, as it is simply $\mathrm{R}_{Q^{*}}$ In a basis where $\hat{r}_{1}$ is taken as the quantization axis, $\mathrm{A}_{\mathrm{Q}}$ is diagonal and we can read off the elgenvalues $E_{m}=w_{Q} \cdot\left[3 m^{2}-I(I+1)\right]$. Then the nuclear angular momentum operators appearing $I_{n} H_{d}$ can be truncated to remove the non-secular elements and calculate $\mathrm{M}_{2}$. ThIs would appear at first to amount to the removal of the nuclear spin components perpendicular to $\vec{r}_{1}$, but in the case of half-1ntegral nuclel the degeneracy of $m= \pm \frac{1}{2}$ allows static 
components of $\vec{I}$ perpendicular to $\vec{r}_{1}$. This calculation is shown in an appenidx, and the result is

$$
M_{2}^{Q}=\frac{1}{3} y_{\mu}^{2} y_{I}^{2} I(I+1) \Sigma I_{i}^{-6}\left[4 \sin ^{2} \theta_{1}+\frac{3(2 I+1)}{\partial I(I+1)}\left(2-\sin ^{2} \theta_{1}\right)\right] \text {. }
$$

This form is correct for half-1ntegral spins; for integral spins the second term in the brackets, which results from the degeneracy, is not present.

It should be noced that if the EFG is due to the presence of the $\mu^{+}$, it w1ll decrease with distance so that the truncation of the nuclear spins will no longer be valid for the higher terms in the sum. However, the relaxation is primarily due to tine first shell of nelghbors, so an error of $<1 \%$ is made by extending the summation over all nuclei.

As mentioned above, expressions quadratic in $\cos \theta$ are 1 sotropic If the $\mu^{+}$has cubtc surroundings, and we see the angular average width

$$
\mathrm{M}_{2}^{Q}(\text { cubIc })=\overrightarrow{\mathrm{M}}_{2}^{Q}=\frac{8}{9} \mathrm{y}_{\mu}^{2} \mathrm{y}^{2} I(I+1) \Sigma \mathrm{r}_{1}^{-6}\left[1+\frac{3(2 I+1)}{16 I(I+1)}\right] \text {. }
$$

In the case of copper, $I=3 / 2$, this reduces to

$$
\begin{aligned}
\vec{M}_{2}^{Q}(I=3 / 2) & =\frac{16}{15} y_{\mu}^{2} y_{I}^{2} I(I+1) \sum_{I} I_{I}^{-6} \\
& =\frac{4}{5} \bar{M}_{2}^{2 f} .
\end{aligned}
$$

Since $M_{2} \propto \Delta_{z}^{2}$, we see that the quadrupole effect is not very large, serving to reduce $\Delta_{z}$ by about $10 \%: \Delta_{z}^{Q / \Delta_{z}}=\sqrt{4 / 5}$. 


\section{EXPERIMENTAL RESULTS}

\section{A. Samples}

Two copper samples have been used in this work. Most of the data have been taken with a sample referred to as the "LBL" copper. This is a slice of a high purity (5-9's, RRR-1800) oxygen annealed single crystal prepared at the National Bureau of Standards, Boulder. ${ }^{47}$ The oxygen annealing was performed in order to precipitate residual 1ron impurities. The orientation is such chat the initial $\mu^{+}$polarization (horizontal) is parallel to a <110> axis and the applied transverse magnetic field (vertical), if any, is parallel to a $\langle 111\rangle$ ax 1 s.

Several runs were also taken on the copper sample used by the CERN group ${ }^{23,25}$ in their transverse-field $\mu^{+}$SR experiments. This is a very high purity (6-9's, RRR 6600) polycrystalline specimen purifled by soluble anode electrolysis and high-vacuum melting. ${ }^{48}$ The 1mpurity content is estimated to be $<20 \mathrm{ppm}$ interstitial (primarily $\mathrm{C}, \mathrm{N}, \mathrm{O})$ and $<1$ ppm substitutional.

\section{B. Preliminary studies}

The first observations of the zero-field relaxation in copper were made in November 1979. This experiment was performed on the M13 beam Ifne at TRIUMF using a provisional $\mu^{+} \mathrm{SR}$ epparatus known as 
"Beaver", which was mounted 1nside a large set of trlaxial Helmholtz coils in order to compensate stray magnetic flelds, primarily due to the beam IIne magnets and the main magnet of the cyclotron.

Data were taken at several temperatures from $40 \mathrm{~K}$ to $160 \mathrm{~K}$ on the LBL copper sample. The data were analyzed by simultaneously fitting the forward and backward spectra to the theoretical curves of Hayano, et al.4 Flrst, a run at $40 \mathrm{~K}$ was analyzed with $V=0$ in order to obtain the values of $\Delta_{z}$ and the asymmetries, then these parameters were held fixed to these values for the rest of the runs. The value found for $\Delta_{z}$ was $0.375 \pm 0.010 \mu \mathrm{s}^{-1}$. The results for $V(T)$ are shown in Table I. Although these data were taken under conditions of severe beam positron background, the slow hopping of the $\mu^{+}$is clearly exh1bited. An Arrhenius plot of these data is shown in Fig. 11, together with the Arrhenlus curve for the best-fit parameters $V_{0}=210 \mathrm{MHz}$ and $Q=900 \mathrm{~K}$. Also shown 1s an Arrhenlus curve using the best known values ${ }^{10}$ obtalned from the transverse-f feld data, $V_{0}=44 \mathrm{MHz}$ and $Q=620 \mathrm{~K}$. The parameters agree in an order-ofmagnitude sense only.

Because of the large backgrounds and $\mu^{+}$I1fetime distortions encountered in tilis run, it is believed that these data are only semi-quantitative1y correct. Due to beam time constraints and the decommissioning of the M9 d.c. separator, it has not been possible to repeat these observations in order to obtain a more rellable measurement of the slow hopping near $100 \mathrm{~K}$. 
During August, 1980, the LBL copper was measured by transverseand zero-field $\mu^{+}$SR at $21.9 \mathrm{X}$ with the Eagle spectrometer on Mg-W3. The static recovery of polarization to $1 / 3$ was clearly seen in these data. Only one set of spectra was taken, and it yielded $\Delta_{z}=0.382 \pm 0.007 \mu^{-1}$ and $\nu=0.05 \pm 0.02 \mu \mathrm{s}^{-1}$. The asymmetr1es were flxed to the values obtalned by a transverse-fleld run for this analysis. In F1g. 15a the data from the B telescope $z z z$ shown with the fit. The asymmetry is obtained from the aw data using the fitted values of $N_{0}$ and $B$. The minimum in the polarization is clearly seen in these data, and the fit to the theory is very good.

\section{c. Measurements Below $5 \underline{\mathrm{K}}$}

The transverse- and zero-field $\mu^{+}$SR measurements were made on the CERN polycrystal and the LBL single crystal copper samples in two

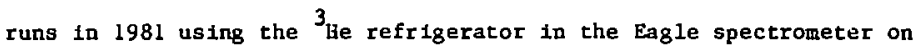
the M9-W3 beam channel. Zero-fleld runs and transverse-fleld runs at 80-100 0e were alternated, and typlcally $10^{7}$ events were taken in each run. Since the shape of the relaxation is of utmost importance In this work, a data gate length of 14-15 $\mu$ sas used in order to have a minimum number of $\mu^{+}$whlch survive longer than the gate length. Data were taken in 8 ns bins out to $12 \mu s$.

The transverse-fleld data were analyzed by fitting to a Gaussian relaxation function with all parameters varlable, and for each of the telescopes $F$ and $B$ weighted averages of the asymmetries were taken over all runs done without disturbing the target. Because the transverse-field data contain oscillations, the asymmetry can be 
determined much more accurately than in zero fleld, where the polarization has a smooth time dependence. As long as the target position and $\mu^{+}$stopping distribution remain constant, the asymetry can be flxed by this method. There were two sets of runs taken, those numbered in the 100's during January, 1981, and those numbered in the $800^{\circ} \mathrm{s}$ during June, 1981. For the January, 1981 data the asymetries were $A_{F}=0.252$ and $A_{B}=0.196$, and for the June, 1981 data the values used were $A_{F}=0.268$ and $A_{B}=0.209$.

The trangverse-fleld data are summarized in Table II. The agreement of the asymmetries is very good among members of each of the two sets of runs. Fig. 12 shows our transverse-fleld widths superimposed on those of Hartmann et al. The agreement is very good; that slightly higher values were obtained by us is probably due to the lower magnetic flelds we used. Their fleld of 520 oe is sufficlent to cause a small reduction in the line width by quenching of the quadrupole interaction (F1g. 10). Also shown 1n F1g. 12 are the CERN data on aluminum, with one point measured in Al during our experiments shown. This shows that any "baseline" relaxation, due to applied fleld inhomogeneity, etc., is simflar between the two experiments.

The zero-field data were analyzed by least-squares fitting to the strong-collision model for $G_{z}\left(\nu, \Delta_{z}, t\right)$. This was done in two stages. First, the curve fits were done with asymmetries taken from the transverse-field data, and both $V$ and $\Delta_{z}$ were allowed to vary. The results for $\Delta_{2}$ are shown in Table III, and F1g. 13 shows $\Delta_{2}$ 
plotted as a function of $T$. From this we conclude that $\Delta_{2}$ is cemperature-1ndependent.

Next, a weighted average of the $\Delta_{2}$ values was taken, ylelding $\Delta_{z}=0.389 \pm 0.003 \mathrm{\mu s}^{-1}$. $\Delta_{\mathrm{z}}$ was flxed to this value, and the fits were done agaln with only $N_{0}, B$, and $D$ varying. The resulting values for $V$ are shown in Table IV.

For these data, we have analyzed the $F$ and $B$ telescopes separately; the discrepancy seen in the $V$ values obtained is several times larger than the statistical error, and we take this as an Indication of the level of systematic error in the measurement of $V$. A plot of $V$ vs. $T$ is shown in F1g. 14. The $F$ and $B$ values are shown connected by a vertical line; the extent of this line above and below the data points shows the statistical error.

Note that two of the runs (ruas 114 and 117 ) near $0.5 \mathrm{~K}$ show values for $V$ which appear to be systematically low. This may be due to some stopping of $\mu^{+}$in liquid helium accumulating in front of the beam since there were some difficulties in the operation of the "He cryostat during the January, 1981 run. We have rejected all runs where the accumulation of ${ }^{4} \mathrm{He}$ was apparent from the behavior of the cryostat, but a small effect on these runs can not be ruled out. Improvements to the cryostat, chiefly the use of ${ }^{3} \mathrm{He}$ as the exchange gas, were made between runs 129 and 132. Th1s completely eliminated this problem, and we are confident that the " $B 00$ " series of runs and the runs from the "l00" serles on the CERN sample represent the best data available. 
Plots of the backward asymmetry for the three " 800 " sertes zero-field runs are shown in Fig. $15 b, c, d$. No significant difference exists between the data at $21.9 \mathrm{x}$ and $5.15 \mathrm{x}$. At $2.35 \mathrm{~K}$ the loss of the recovery of polarization 1 complete, and at $0.63 \mathrm{~K}$ the onset of motional narrowing is easily visible.

The agreement between the polycrystal and the single crystal samples is good for both $V$ and $\Delta_{z}$. The equality of $\Delta_{z}$ can be taken as evidence for the octahedral location of the $\mu^{+}$since, as mentioned above, the zero-field width is isotropic only for a site with cublc symmetry.

We have used the Gaussian relaxation function in fitting the transverse-field data shor in Table II In order to compare our data with those of Hartmann, et al. However, since the zero-field work clearly shows a temperature-1ndependent width, a more proper analysis would be to hold the width constant and use Eq. III.11 to get the hopping rate $V$ directly. Th1s has been done for the " 800 " series of runs, and the results are shown In Table $V$ and $F 1_{g} \cdot 16$, along with the hopping rates deduced frow the zero-field method using a simulcaneous forward-backward $f \pm t$.

There is a clear discrepancy between the hopping rates given by the two methods. The reason for this is that the transverse-field data were taken at a fleld not sufficlently high to completely average the non-seciiar terms. Since these terms are then varying on a tIme comparable to the $\mu^{+}$relaxation rate, their effect is to create a fictilious addition to the hopping rate, which 1 , after all, 
really a measure of the fluctuation times of the magnetic fields serin by the $\mu^{+}$.

A quantitative calculation of this additional "pseudo-hopplag" 1s quite difficult as it requires a solution to the equation of motion for the density matrix of the $\mu^{+}$spin and 1 ts nearest-na1ghbor nuclear spins. This treatment must also include the quadrupolar Interaction, and enhancements of the relaxation may occur due to this as the quadrupolar level-splitting is near the $\mu^{+}$Zeeman splitting, leading to the possibility of quadrupole-induced muon spin-filps.

We have not attempted to perform these calculations, as the primary purposes for our takıng transverse-field data were to provide the Instrumental asymmetries for use in the zero-field analysis and to compare it with the CERN data. In any case, the hopping rate clearly Increases as the temperature is lowered. 
CHAPTER V

\section{DISCUSSION AND SUMMARY}

\section{A. The Did rolar Width}

Copper is an fcc metal with a cubic lattice constant of $3.61 \AA$ and two abundant 1sotopes, ${ }^{63} \mathrm{Cu}(69.1 \%)$ and ${ }^{65} \mathrm{Cu}(30.9 \%)$. Both have nuclear spin $3 / 2$ with similar gyromagnetic ratios and quadrupole moments, $y\left({ }^{63} \mathrm{Cu}\right)=2 \pi \times 1.1285 \mathrm{kHz} / 0 \mathrm{e}, y\left({ }^{65} \mathrm{Cu}\right)=2 \pi \times 1.2090 \mathrm{kHz} / 0 \mathrm{e}$, and $Q\left({ }^{63} \mathrm{Cu},{ }^{65} \mathrm{Cu}\right) \equiv-0.15 \times 10^{-24} \mathrm{~cm}^{2}$. For calculations of the widths, the proper average of $y$ is the weighted mean square value, $y_{\text {eff }}=2 n \times 1.154 \mathrm{kHz} / 0 \mathrm{e}$, and we use this value $1 \mathrm{n}$ all work.

The theory presented in Chaptin III shows that gtven these parameters, relaxation rates can be computed from first principles with only one adjustable parameter--the expansion of the nearest nelghbors of the $\mu^{+}$. Once this 1 s known we can calculate the sums in Eqs. III.5 and III. 6 to obtain the zero-fie.ld and high-field widths. Transverse-field experiments ${ }^{7-10}$ have provided us with this parameter. The best value available is $4.9 \%$, given in Ref. 10 with no error quoted. An estimate $o i$ the error of this can be taken from Ref. 8, where the value $\sigma_{\mathrm{vV}} / \sqrt{2}=0.2589 \pm 0.0036 \mu^{-1}$ was quoted for the high-field relaxation rate for the <100> direction at 4500 0e. This is in reasonable agreement with the value of $0.265 \mu^{-1}$ calculated from Eq. III.5 with a 4.9\% nearest-neighbor expansion. When this is used In Eq. III.12 we obtain the expected value 


$$
\Delta_{z} \text { (theory) }=0.350 \pm 0.005 \mu \mathrm{s}^{-1} \text {, }
$$

whereas the data presented in chapter IV showed that

$$
\Delta_{\mathrm{z}} \text { (experiment) }=0.389 \pm 0.003 \mu^{-1} \text {. }
$$

Note that the criterion for validity of truncating the nuclear spins 1s well sat1sfled, $\Delta_{z} / 6 w_{Q}=0.06$.

The value of $\Delta_{2}$ and 1 ts error are taken from a welghted average of measurements at different temperatures. At any specific temperature, the statistical error of $\Delta_{2}$ is typically $\pm 0.010 \mu \mathrm{s}^{-1}$.

As mentioned in chapter III, the zero-field width is isotropic if the $\mu^{+}$site has cubic symmetry. The good agreement of single crystaj. and polycrystalline samples is evidence that the site is cubic at all temperatures studiel, but a more sensitive test would be a measurement of the orfentation dependence of the width in a siagle crystal.

It should also be pointed out here that an earlier measurement of copper in near-zero magnetic fields ${ }^{49}$ yielded a similar value of $\Delta_{z}=0.372 \pm 0.004{\mu s^{-1}}$, although the use of an incorrect fitting function and an incorrect calculation of the quadrupole effect on the width hindered the interpretation of this result.

We now consider several possible explanations for the disagreement between the transverse-fleld and zero-field dipolar widths.

(1) The EFG may not be in the radial direction and/or the quadrupole interaction may not be axtally symmetric. 
(2) The lattice distortion about the $\mu^{+}$may not have cubic symmetry.

(3) The $\mu^{+}$is not actualiy a point particle; rather, 1 ts wave function has a finte extension about the center of the 0 site.

(4) The EFG may be temperature dependent, leading to a breakdown of truncation at low temperatures. A $\mathbf{T}^{3 / 2}$ temperature dependeace has been suggested. 50

No estimates have been made of the first two effects, but we should note that these are related by a point first proposed in Ref. 8. There it was suggested that the lattice distortisn and resulting EFG were not due solely to the self-trapping distortions caused by the $\mu^{+}$, but rather that the $\mu^{+}$are being trapped at energetically favorable sites in the strain field of a lattice imperfection. There would then exist a pre-formed EFG and lattice distortion, which would not be symmetric about the $\mu^{+}$, and the simple theory we have used would be only approximate. The present experiment has show consistent values of $\Delta_{z}$ for both single crystal and polycrystal specimens, which would indicate a limit on the deviation from cubic symmetry of the expansion.

Anomalies in the low fleld relaxation at low temperatures are seen in the transverse-field data of several workers in copper and other metals. These have been attributed to non-radial ${ }^{10}$ or temperature-dependent 51 EFGs. No satisfactory understanding of this behayior exists, so it is 11kely that departure of the behavior of the EIG from the simple assumptions previously made can contribure to the deviation of $\Delta_{z}$ from the expected value. 
If the $\mu^{+}$has a wave function $\psi(\vec{r})$, then its position must be averaged over to obtain the dipolar Hamfltonian,

$$
H_{d} \rightarrow \int A_{d}(\vec{r})|\psi(\vec{r})|^{2} d^{3} \vec{r} \text {. }
$$

No calculations exist which give the effect on the magnitude and 1sotropy of $\Delta_{z}$ due to the spread of the $\mu^{+}$ground state wave function. It has been shown ${ }^{41}$ that if one assumes a spherical wave function, $|\psi(\vec{r})|^{2} \propto \exp \left(-r^{2} / 2 a^{2}\right)$, the spread of the wave function will serve to decrease the value of $B_{d}$ Isotropically by a factor $\lambda(r / a)$ whtch approaches unity rapidly for $r>a$. S1nce all components of the auclear dipole field at the $\mu^{+}$are decreased by $\lambda$, this cannot affect the transverse-field and zero-fleld widths differently.

$\operatorname{Th} 1 \mathrm{~s}$, as well as a lattice expansion about the $\mu^{+}$, could thus be responsible for the decreased relaxation rates observed. However, the ground state wave function would not be spherical, so due to the different angular dependences of the zero-field and transverse-field widths, the reduction of the width could be different in the two cases, and could give an anisotropy in the zero-field width.

To estimate the size of this effect would require realistic wave functions for the $\mu^{+}$, but we can note that $\lambda$ increases rapidly from $\lambda=0.5$ at $a=1 / 2 r_{n n}$ to $\lambda=0.85$ at $a=1 / 3 r_{n n}$. In Cu these numbers are $0.9 \AA$ and $0.6 \AA$ respectively. If an anisotropy in the correction factor is to account for the difference in the transverse-field and zero-field widths, then we tust have a spreading of the wave function of at least $0.6 \AA$. This could not be ruled out on the basis of the 
potential energy curve given In Ref. 19 (FIg. 2).

It is likeiy that the extension of the ground state wave function at the $\mu^{+}$site is responsible for at least some of the reduction In $\sigma_{V V}$ from the point-muon, unrelaxed-lattice value. This is also Indicated theoretically, as the calculated value ${ }^{19}$ of 0.03 for the nearest nelghbor expansion is smaller than the 0.05 inferred from the experiments.

Note that the entire deviation of $\sigma$ from its value for an unrelaxed lattice could be accounted for by a $\mu^{+}$wave function of with $a=0.6 \AA$ even in the absence of a lattice expansion. 41

B. Low Temperature Diffusion and Trapping

The second principal aspect of this work is the use of the zero-field $\mu^{+} S R$ method as a complement to the transverse-field method In determining the $\mu^{+}$mobllity. He are able to clearly demonstrate that the reduction of the transverse-field line width seen by others is due to hopping of the $\mu^{+}$in a distribution of random local fields having a constant width. Quite apart from the magnitude of $\Delta_{2}$, its temperature Independence and orlentation Iadependence (1nferred from the equivalence of single crystal and polycrystalline samples) provides Evidence that the $\mu^{+}$moves among equivalent octahedral sites rather than migrating to different species of trapping sites at different temperatures.

Our experiments do not, however, give a clear indication of the gechanism of d.tfusion below $2 \mathrm{k}$. The theory of light-interstitial 
diffusion has recelved a great deal of attention, $16-22,52-61$ and a variety of opinion ${ }^{62-66}$ extsts as to the applications of the theory to understanding the experiments on $\mu^{+}$motion at low temperatures. We do not intend to present a comprehensive discussion of this subject here, but rather to touch on a few points of interest to the present case.

One of the central questions in the study of $\mu^{+}$SR in metals is the possibllity of observing the coherent motion of the $\mu^{+}$, $1 . e$. the Inciplent formation of a delocalized Bloch wave which would allow the $\mu^{+}$to propagate rapidly by tunneling through the lattice. This mechantsm would show a decreasing hopping rate with increasing temperature because of the disruption of the narrow $\mu^{+}$energy band by phonons, but estimates of the temperature above which coherent tunneling should be negligible range from $10^{-17} \mathrm{~K}$ to $200 \mathrm{k} .^{63}$ Even if a completely delocalized wave cannot form due to lattice Imperfections, local tunneling states whth a spreading of the $\mu^{+}$wave function over a few adjacent sites may occur, and may actually be enhanced by the presence of defects.

However, even in a materlal that exhlbits an iritrinsic diffusion rate that Increases with temperature, the presence of traps for the muons (for tnstance impurities or lattice 1mperfections) can result In an apparent decrease in the hopping rate as the temperature is increasti. 67,68 a schematic transverse-field depolarization curve representing a crystal with a single species of trap is shown 1n FIg. 17. At very low temperatures, the intrinsic diffusion rate is small 
and the depolarization is characteristic of the self-trapped state In the pure material. As the temperature 1s raised, the muons begin to diffuse faster, showlng motional narrowing, and then begin to find traps, which causes again a strong locallzation and a corresponding high relaxation rate. The relaxation rate then remains roughly the same unt1l a temperature is reached such that the muons can be thermally detrapped, and the relaxation curve once again approaches that for the pure matertal.

The relaxation curve of FIg. 17 is characterized by two temperatures, a trapplng temperature $T_{t}$ depending on the trap concentration and a detrapping temperature $T_{d}$ proportional to the activation energy required to escape the traps. As shown In FIg. 17, the effect of increasing the trap concentration is to lower $T_{t}$ thus decreasing the depth of the dip in the relaxation rate. Such behavior is in fact seen $10 \mathrm{~N}$-doped $\mathrm{Nb}^{69}$ where $\mathrm{T}_{t}-20 \mathrm{~K}$ and $\mathrm{T}_{\mathrm{d}}-80 \mathrm{~K}$.

If such a trappling model were invoked to explain the decrease of $\sigma$ below $2 \mathrm{~K}$ in copper, the concentration of traps would need to be very high, since our zero-fleld data clearly show hopplng rates $\leq 0.5 \mu \mathrm{s}^{-1}$, Indlcating that the traps must be found within only a few hops.

Petzinger ${ }^{70,31}$ has shown that the zero-field method has a unique ability to distingulsh trap-1imited diffusion from 1ntrinsic diffuston. In a simple model where the intrinsic diffusion is assumed rapid enough that depolarization occurs only at traps and where no detrappling occurs, he has calculated $G_{z}\left(r, \Delta_{z}, t\right)(F / g, 18)$, where $r$ 
is the rate at which the $\mu^{+}$distribution relaxes to the traps. This should apply to the zero-field spin relaxation in the region where $\sigma$ shows an upward slope toward the defect-trapped state in Fig * 17.

Our data clearly contradict his result. The trap-limited $G_{z}\left(r, \Delta_{z}, t\right)$, like the Kubo-Toyabe function, shows a minimum in the polarization (for large enough $r$ ) but always recovers to an asymptotic value of $1 / 3$. For $r \rightarrow \infty$ the static Kubo-Toyabe function, Eq. III. 3, is recovered, but for slower trapping rates the minimum in $G_{z}(t)$ is lost and the polarization decays monotonically. Furthermore, the inftial decay rate in this model shows large changes with $r$ even at earlier times than at the minimum, whereas our data are clearly consistent with the Kubo-Toyabe theory where the inftial decay rate is constant until the hopplng becomes suffictently rapid to cause a complete itzo af the recovery of the polarization to $1 / 3$. This indicates that the $\mu^{+}$experiences more than one non-zero, stati: value of the random magnetic field during the observation time.

A complete theory of relaxation/trapping would have to account for both relaxation at traps as well as relaxation whlle undergoing Intrinsic diffusion between traps. However, since our data are well described by the Kubo-Toyabe model, we have no evidence for traplimited diffusion in copper.

As noted by several authors, $25,63,71$ the results obtained for $\sigma(T)$ lil copper are remarkably insensitive to the purfty of the material. The slope of o(T) near $150 \mathrm{~K}$ is similar in samples of very different purity, and our data show good agreement between two 
samples of different purity in their behavior below $5 \mathrm{~K}$. This ind1cates that if the trapping of the $\mu^{+}$is due to 1mpirities, then they are present in high concentration in all copper samples.

One possible source of a high concentration of weak traps, small Inhomogeneities In the lattice behavior due to the 1sotopic mixture of natural copper, has been ruled out by experiments ${ }^{63}$ on isotopically pure $(99.6 \%){ }^{63} \mathrm{Cu}$. These measurements have shown s.o significant differences from natural copper. Although one cannot completejy rule out trapping at the remainting concentration of ${ }^{65} \mathrm{Cu}$ or at chentcal impurities, trapping by this mechanism seems to be unlikely in view of these observations.

Another potential source of traps is the presence of long range elastic strain fields ${ }^{63,72}$ caused by interstitial impurities $(C, N, 0)$ in the crystal. A single impurity can facilitate trapping at many sites by this mechanism, so the concentration does not need to be high. (Impurfties at the $100 \mathrm{ppm}$ level can create strain fields throughout the lattice ${ }^{73}$ which are sufficient to sause localization by the Anderson ${ }^{74}$ mechanlsm.) We should also note that calculations 75 have show that the strain fields may actually catalyze self-trapping ar favored sites at the end of the muon thermalfzation process. If this were the case, the zero-field relaxation would not be expected to follow the theory of Petzinger, since the inftial distribution of muons through the crystal would not be unfform. Instead, the muons would all be trapped essentially at $t=0$. 
In summary, the high concentration of traps that would be required to give trap-limited diffusion at $5 \mathrm{~K}$ taken together with the sample purity independence of the relaxation behavior and the gooũ agreement with the Kubo-Toyabe relaxation functions in zero field are all consistent with intrinsic diffusion in a pure copper host crystal. We cannot, however, rule out the posstble role of long range strain fields as traps or trap-enhancers. Since there would be a distribution of trap energies and concentrations in that case, che simple model of Petzinger would be Inadequate, and a Kubo-Toyabe behavior could be seen as the muons become trapped by deeper traps occurring in lower concentrations as the temperature is increased above $0.5 \mathrm{~K}$.

\section{Future Work}

The study of the diffuston of the $\mu^{+}$through its spin relaxation is one of the oldest problems in modern $\mu^{+} S R$ research, and the development of the zero-field method has opened up new avenues of Inquiry in this as well as other problems. In this work, we have by no means exhausted the possibilities for zero-field studies in copper. When the d.c. separator is recommissioned on the improved M20 channel, zero-field $\mu^{+} \mathrm{SR}$ w11 agaln be feastble at TRIUMF, and several possibilities are suggested for further experiments.

F1rst, a new measurement of the hopping rate in the range of $80 \mathrm{~K}$ to $150 \mathrm{~K}$ would be very valuable, espectally in the slow hopping region inaccessible to transverse-field $\mu^{+}$SR. This may yield Important details about the validity of the quantum diffusion theory $16-22$ 
in this region, where so far the agreement with experiment 9,10 is very good. It may be possible to see further deviations from the Arrhenius behavior due to the temperature-dependent prefactor and activation energy given by the theory.

Second, the 1sotropy of $\Delta_{z}$ should be carefully studied in the region of $10 \mathrm{~K}$ to $80 \mathrm{~K}$ where minimum hopping exists. As we have shown, this w11 be sensitive to deviations from cublc symmetry of the lattice distortions and EFGs occurring about the $\mu^{+}$and/or spreading of the $\mu^{+}$wave function. This may yield important clues as to the rature of the trapped state of the $\mu^{+}, 1 . e$. whether one sees self-trapplng in the pure material or inpurity-enhanced trapping In a pre-exiating lattice deformation.

Third, the measurements should be extended to temperatures as low as posstble. The transverse-field experimeats show a plateau in $\sigma$ below $0.5 \mathrm{k}$, but the sensitfvity of the zero-field method to slow hopping may uncover structure which has gone heratofore unnoticed. 
APPENDIX A

Zero-field Second Moments

The second moment can be calculated as

$$
\mathrm{M}_{2}=-\frac{\operatorname{tr}\left\{\left[\mathrm{H}_{\mathrm{d}}, \mathrm{s}_{\mathrm{z}}\right]^{2}\right\}}{\operatorname{tr}\left[\mathrm{s}_{\mathrm{z}}^{2}\right]}
$$

We take the 2 axis as the initial $\mu^{+} \operatorname{spln} d$ drection. $H_{d}{ }^{\prime}$ is the "truncated" dipolar Hamiltonian defined in the bas is of the unper-

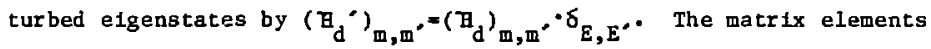
of $\mathrm{H}_{\mathrm{d}}$ which connect states of different unperturbed energy must be dropped. As argued In chapter III, these elements sreate separate lines rather than broadening the main line if their frequencies are far enough from the main IIne. We will drop the prime from $\mathrm{H}_{\mathrm{d}}$ In what follows, it being understood that we wlll truncate it appropriately.

$H_{d}$ is properly written as a sum over all nuciear dipoles, with the traces taken over the $(2 I+1)^{N}(2 s+1)$ states or the muon-nuclel system. But since the traces of terms containing products of operators for dffferent nuclei vantsh, we cust calculate the moment for each nucleus individually and add the contributions when finished.

The calculation is most conveniently carried out in cooldinates $\langle x, y, z)$ where the $z$ axis is along the radial direction for each nucleus. We then have 


$$
{ }_{z}=s_{z} \cos \theta-s_{x} \sin \theta
$$

and

$$
B_{d}=y_{\mu} y_{I^{I}}{ }^{-3}\left[-2 s_{z} I_{z}+1 / 2\left(s_{+} I_{-}+s_{-} I_{+}\right)\right]
$$

Calling the operator in square brackets $K$, the numerator of equation A. 1 is proportional to $\operatorname{tr}\left\{\left[K,\left(s_{z} \cos \theta-s_{x} \sin \theta\right)\right]^{2}\right\}$. We have

$$
\begin{aligned}
& {\left[K, s_{z}\right]=1\left[s_{x} I_{y}-s_{y} I_{x}\right] \text { and }\left[K, s_{x}\right]=-1\left[2 s_{y} I_{z}+s_{z} I_{y}\right] \text {, so }} \\
& -\left[K, s_{z}\right]^{2} \rightarrow \cos ^{2} \theta \cdot\left[s_{x} I_{y}-s_{y} I_{x}\right]^{2}+\sin ^{2} \theta \cdot\left[2 s_{y} I_{z}+s_{z} I_{y}\right]^{2} \\
& \rightarrow \cos ^{2} \theta \cdot\left[s_{x}^{2} I_{y}^{2}+s_{y}^{2} I_{x}^{2}\right]+\sin ^{2} \theta \cdot\left[4 s_{y}^{2} I_{z}^{2}+s_{z}^{2} I_{y}^{2}\right] \text {. }
\end{aligned}
$$

All traceless terms such as $s_{x} s_{y}$ have been dropped. The traces of the $\mu^{+}$spin operators can now be eliminared. Remembering that the traces extend over the auclear spin coordinates as well, we have

$$
M_{2}=\frac{y_{\mu}^{2} y_{I}^{2} r^{-6}}{(2 I+1)} \cdot\left\{\cos ^{2} \theta\left[t r I^{2}+t r I^{2} I^{2}\right]+\sin ^{2} \theta\left[4 t r_{I} I_{z}^{2}+t r_{I} I_{y}^{2}\right]\right\}
$$

The notation $t r_{I}$ indicates a trace over only the nuclear spin coordinates. To proceed further, we must specify the truncation to be performed on $\vec{I}$.

\section{Pure dipolar case}

If there are no quadrupole moments, the unperturbed Hamiltonian is absent and we use the full operator $\vec{I}$ in equation A.2. For any component of $\vec{I}, \operatorname{tr}_{I} I_{j}^{2}=(1 / 3) I(I+1)(2 I+1)$, and summing over all nuclei we have

$$
M_{2}=\frac{1}{3} y_{\mu}^{2} y_{I}^{2} I(I+1) \bar{\Sigma} I_{1}^{-6}\left[5-3 \cos ^{2} \theta_{1}\right]
$$




\section{Quadrupole case}

If there is a quadrupole interaction of sufficient strength that its energy level spacings greatly exceed the line width, then we must remove all matrix elements of $\vec{I}$ between different energies. The quadrupole Hamiltonlan is

$$
\mathrm{B}_{\mathrm{Q}}=\mathrm{u}_{\mathrm{Q}} \cdot\left[3 \mathrm{I}^{2}, \mathrm{I}(\mathrm{I}+1)\right] \text {, }
$$

and we see $t \cdot t$ the elgenvalues are given by $E_{m}=w_{Q} \cdot\left[3 m^{2}-I(I+1)\right]$. Now $\vec{I}$ has non-vantshing matrix elements $\vec{I}_{m, m^{\prime}}$ for $m^{\prime}=m^{\prime}$ and $m=m^{\circ} \pm 1$, and the only states for which these are degenerate are $m= \pm 1 / 2$. The proper truncation is then:

(1) for Integral spins, drop $I_{x}$ and $I_{y}$ completely, and

(2) Eor half-integral spins, drop al1 but the mmt $1 / 2$ elements of $I_{x}$ and $I_{y}$.

The Integral (I) case can now be disposed of Immediately,

$$
M_{2}^{Q}(I)=\frac{4}{3} y_{\mu}^{2} y_{I}^{2} I(I+1) \Sigma_{i} I_{i}^{-6} s I n^{2} \theta_{1} \text {. }
$$

For the half-integral (HI) case, we have the truncated operators

$$
I_{x, y}=\frac{2 I+1}{4}\left[\begin{array}{lll}
0 & & \\
& \sigma_{x, y} & \\
& & 0
\end{array}\right] \text {, }
$$

where $\vec{\sigma}$ represents the Paull matrices acting on the $m= \pm 1 / 2$ states. From this we see that $\operatorname{tr}_{I}\left(I^{\prime}{ }_{x}\right)^{2}=\operatorname{tr}_{I}\left(I^{\prime}{ }_{y}\right)^{2}=\frac{1}{8}(2 I+I)^{2}$, and we get ${ }^{4}$

$$
M_{2}^{Q}(H I)=\frac{1}{3} y_{\mu}^{2} y_{I}^{2} I(I+I) \Sigma r_{1}^{-6}\left[4 \sin ^{2} \theta_{1}+\frac{3(2 I+1)}{8 I(I+1)}\left(2-\sin ^{2} \theta_{1}\right)\right] \text {. }
$$


Acknowledgements

Experiments of this nature are by necessity the work of more than one person. For this reason, the "we" that appears in the text is usually intended as a plural pronoun and not as an editorial device. I would like to thank all the principal collaborators on the low temperature copper experimenc--Ken Crowe, Steve Rosenblum, Jess Brewer, Chao-Yuan Huang, Jim Smlth, and Stan Kohn. In particular, Chao-Yuan Huang, Jim Smith, Stan Kohn, and Steve Rosenblum performed most of the work of designing, fabricating, and testing the hellum-3 refrigerator, and Jess Brewer and Steve Rosenblum performed much of the data analysis. These individuals have also contributed greatly to our other $\mu^{+} \mathrm{SR}$ efforts at TRIUME.

Speclal thanks must go to Ken Crowe, who as group leader has maintained a strong effort in $\mu^{+}$SR even in the face of an uncertain funding situation.

I also extend my thanks to Dave Spencer, Dale Harshman, Don Fleming, Dave Garner, Rob Kiefl, Hugo Schilling, and Alex Schenck, who have, at one time or another, provided manpower and expertise when needed on the experimental runs. Rudi Abegg provided valuable assistance in the maintenance and operation of the helium-3 cryostat.

Tomo Uemura is thanked for helpful discusstons and for stimulatIng my early interest in the problem, as well as for collaboration on the zero-field measurements at higher temperatures and for kindly 
providing me with the results of his numerical computations of the zero-field relaxation function. I have also benefited from discusslons of zero-field relaxation with Rolf Keltel, BIll Zajc, and Jim Bistirlich in addition to the principal collaborators mentioned above.

I am most grateful to Er Ik Karlsson and Alain Yaouanc for theì generous permission to cut a sample trom thelr copper polycrystal, and to Fred Flckett for supplylng the copper crystal. Roy Bossingham oriented and cut the copper samples.

I would also like to thank the entire staff of TRIUMF for their efforts in delivering the beam to the experimenters and for the many other services which are necessary when performing work as visitors at the1r laboratory.

Finally, I would like to thank my famfly for encouragling my Interest in science from an early age and for providing financlal support during my undergraduate education.

Th1s work was supported in part by the taxpayers of the Untted States through U.S. Department of Energy contracts DE-ACO3-76SF0009 Id AT03-81ER40004. 


\section{REFERENCES}

1. J.H. Brewer, K.M. Crowe, F.N. Gygax and A. Schenck, 1n Muon Physics edited by Vernon W. Hughes and C.S. Wu, Vol. III, (Academ1c, New York, 1975).

2. J.H. Brewer and K.M. Crowe, Ann. Rev. Nucl. Part. Sc1. 28 (1978) 239.

3. A. Scheack, in Nuclear and Particle Physics at Intermediate Energles edited by J.B. Warren, (Plenum, New York, 1976).

4. R.S. Hayano, Y.J. Jemura, J. Imazato, N. N1sh1da, T. Yamazaki and R. Kubo, Phys. Rev. B20 (1979) 850.

5. I.I. Gurevich, E.t. Mel'eshko, I.A. Muratova, B.A. Nikol'skil, V.S.Roganov, V.I. Selivanov and B.V. Sokolov, Phys. Lett. 40 s (1972) 13.

6. V.G. Grebinnik, I.I. Gurevich, V.A. Zhukov, A.P. Manych, E.A. Mel'eshko, I.A. Muratova, B.A. N1kol'skil, V.I. Selivanov and V.A. Suet1n, So.. Phys.-JETP 41 (1976) 777 [2h. Eksp. Teor. F12. 68 (1975) 1548].

7. M. Camani, 2.N. Gygax, W. Ruegg, A. Schenck and H. Schilling, Phys. Rev. Lett. 39 (1977) 836.

8. M. Camant, D.G. Fleming, F.N. Gygax, W. Ruegg, A. Schenck and H. Schilling, Hyperfine Int. $\underline{6}$ (1979) 265. 
9. H. Schiliting, Ph.D. Thesis, no. 5608, ETH Zurich (1980).

10. H. Sch1lling, M. Camant, F.N. Gygax, W. Ruegg, and A. Schenck, Hyperfine Int. $\underline{8}$ (1981) 675 .

11. 0. Hartmann, Phys. Rev. Lett. 39 (1977) 832.

12. A. Abragam, The Princ1ples of Nuclear Magnet1sm, (Oxford, London, 1961), chapter X.

13. R. Kubo and K. Tomita, J. Phys. Soc. Japan 9 (1954) 888.

14. J.H. Van Vleck, Phys. Rev. 74 (1948) 1168.

15. 0. Hartmann, E. Karlsson, K. Pernestal, M. Borghinf, T.o. N1fulkoski and L.0. Norl1n, Phys. Lett. 6lA (1977) 141.

16. H. Telchler, Phys. Lett. 6쏘 (1977) 78.

17. H. Tetchler, Phys. Lett. 67A (1978) 313.

18. H. Telchler, Hyperfine Int. 6 (1979) 251.

19. H. Te1chler, 1n Exotic Atoms '79, Fundamental Interactions and Structure of Matcer, edited by K. Crowe, J. Duclos, G. Fiorentini and G. Torelli (Plenum, New York, 1980), p. 283.

20. H. Teichler, Byperfine Int. $\underline{8}$ (1981) 505 .

21. C.P. Flynn and A.M. Stoneham, Phys. Rev. B1 (1970) 3966.

22. Yu. Kagan and M.I. Klinger, J. Phys. C? (1974) 2791.

23. 0. Hartmann, E. Karlsson, L.0. Norlin, T.O. N1inikoski, K. H. Kehr, D. Richter, J.-M. Welter, A. Yaouanc and J. Le Hericy, Phys. Rev. Lett. 44 (1980) 337. 
24. 0. Hartmann, Hyperfine Int. $\underline{8}$ (1981) 525.

25. 0. Hartmann, L. O. Norlin, A. Yaouanc, J. Le Hericy, E. Karlsson and T.0. NiIntkoskt, Hyperfine Int. $\underline{8}$ (1981) 533.

26. C.H. Clawson, K.M. Crowe, S.E. Koha, S.S. Rosenblum, C.Y. Huang, J.L. SmIth and J.H. Brewer, Proceedings of LT-16, to be published in Physica (1982).

27. A.E. P1fer, T. Bowen and K.R. Kendall, Nuci. Instrun. Methods 135 (1976) 39.

28. C.J. Oram, J.B. Warren, G.M. Marsha1l and J. Doornbos, Nucl. Instrum. Methods 179 (198I) 95.

29. D.M. Garner, Ph.D. Thesis, Dept, of Chemistry, Univ. of British Columbia, 1979.

30. Avallable from the CERN program library, Division DD, CERN, CH 1211, Geneve 23, Switzerland.

31. T. Yamazak1, Hyperfine Int. $\underline{6}$ (1979) 115.

32. Y.J. Uemura, R.S. Hayano, J. Imazato, N. NIshida and T. Yamazak1, Solld State Comm. $\underline{31}$ (1979) 731 .

33. We neglect the Electron splne fut the metal. They serve to create a small knight shift of the muon precession frequency and a small Korringa relaxation.

34. J. Owen, M.E. Browne, H.D. Knight and C. Kittel, Phys. Rev. 102 (1956) 1501 . 
35. Abragam (op. c1t., chapter IV) gives this in the form $\left\langle s_{x}(t)\right\rangle \propto \operatorname{tr} s_{x} s_{x}(t)$, from which our form for $\left\langle s_{+}(t)\right\rangle$ may be easily derived.

36. I. J. Lowe and R.E. Norberg, Phys. Rev. 107 (1957) 43.

37. A. Abragam, op. cit., chapter IV.

38. R. Kubo and T. Toyabe, in Magnetic Resonance and Relaxation, edited by R. Blinc, (North Holland, Amsterdam, 1967).

39. R.E. Halstedt and L.R. Walker, Phys. Rev. Bg (1974) 4857.

40. R. Kubo, Hyperfine Int. 8 (1981) 731.

41. T. McMullen and E. Zaremba, Phys. Rev. B18 (1978) 3026.

42. P. H. Anderson and P.R. Welss, Rev. Kod. Phys. 25 (1953) 269.

43. Y.J. Uemura, M.S. Thes1s, Department of Physics, University of Tokyo, 1979.

44. H.A. Zajc, private communication.

45. J.H. Brewer, private communication.

46. A.T. Fiory, Hyperfine Int. $\underline{8}$ (1981) 777.

47. F. F1ckett, Mat. Sc1. Eng. 14 (1974) 199.

48. H. Bruning and J. Le Hericy, to be published.

49. V.G. Grebinnik, I.I. Gurevich, A.I. Klimov, V.N. Majorov, A.P. Manych, E.V. Mel'nikov, B.A. Nikol'skil, A.V. Pirogov, A.N. Ponomarev, V.I. Selivanov, V.A. Suetin and V.A. Zhukov, Hyperfine Int. 6 (1979) 275 . 
50. 0. Hartmann, E. Karlsson, L.0. Norlin, D. Richter, and T.0. N11nikosk1, Hyperfine Int. $\underline{6}$ (1979) 289.

51. J.H. Brewer, E. Koster, A. Schenck, H. Schilling, and D.L. W11liams, Hyperfine Int. $\underline{8}$ (1981) 671 .

52. A.M. Stoneham, Hyperfine Int. $\underline{6}$ (1979) 211 ,

53. A.M. Stoneham, 1n Exottc Atoms '79, Fundamental Interactions and Structure of Matter, edited by K. Crowe, J. Duclos, G. Florentinl and G. Torelli (Plenum, New York, 1980), p. 209.

54. D. Emin, Phys. Rev. Lett. $\underline{25}$ (1970) 1751.

55. D. Emin, Phys. Rev. B.3 (1971) 1321.

56. Dav1d Emin, M.I. Baskes, and W.D. Wilson, Phys. Rev. Lett. 42 (1979) 791.

57. A. Yaouanc, Phys. Lett. 87A (1982) 423.

58. T. McMullen and B. Bergergon, Solid State Comm. 28 (1978) 31.

59. D. RAchtet, In Exotic Atoms ' 79 , Eundamental Interactions and Structure of Matter, edited by K. Crowe, J. Duclos, G. Florentin1 and G. Torelli (Plenum, New York, 1980), P. 245.

60. S. Fuf 11 and Y.J. Uemura, Sol1d State Comm. 26 (1978) 761.

61. S. Fuj11, J. Phys. Soc. Japan 46 (1979) 1833.

62. V.G. Grebinntk, I.I. Gurev1ch, V.A. Zhukov, A.I. Klimov, V.N. Majorov, A.P. Manych, E. V. Mel'nikov, B.A. N1kol'skil, A.V. Pirogov, A.N. Ponomarev, V.I. Selivanov and V.A. Suetin, PIsma 2h. Eksp. Teor. Fiz. $\underline{25}$ (1977) 322 . 
63. E. Karlsson, Hyperfine Int. $\underline{8}$ (1981) 647.

64. H. Graf, G. Balzer, E. Recknagel, A. Weldinger and R.I. Grynszpan, Phys * Rev. Lett. 44 (1980) 1333.

65. K.W. Kehr, D. Richter, J.M. Helter, O. Hartmann, L.O. Norlin, E. Karlsson, T.0. N11nikoskf, J. Chappert and A. Yaouanc, Hyperfine Int. $\underline{8}$ (1981) 681 .

66. David Emin, Hyperfine Int. $\underline{8}$ (1981) 515.

67. K.G. Petzinger, R.L. Munjal, and E. Zaremba, Hyperfine Int. $\underline{6}$ (1979) 223.

68. K. H. Kehr, D. Richter, and G. Honig, Hyperfine Int. $\underline{6}$ (1979) 219.

69. T.0. N11nikosk1, 0. Hartmann, E. Karlsson, L. -0. Norlin, K. Pernestal, K.W. Kehr, D. Richter, E. Walker, and K. Schulze, Hyperfine Int. $\underline{6}$ (1979) 229.

70. K.G. Petzinger, Phys. Lett. 75A (1980) 225.

71. K.G, PetzInger, Hyperfine Int. $\underline{8}$ (1981) 639.

72. E. Karlsson, in Exotic Atoms '79, Fundamental Interactions and Structure of Matter, edited by K. Crowe, J. Duclos, G. Florentinl and G. Torell1 (Plenum, New York, 1980), p. 303.

73. O. Hartmann, E. Karlsson, L.O. Norlin, D. Richter and T.O. N11n1kosk1, Phys. Rev. Lett. 41 (1978) 1055.

74. P.W. Anderson, Phys. Rev. $\underline{109}$ (1958) 1492. 
75. A. Browne and A.M. Stoneham, unpublished. (Preprint in $\mu$ SR Newsletter 23 (1979) 1060 . 
Table I

Zero-field Hopping Rate $100-160 \mathrm{~K}$

\begin{tabular}{lc}
\hline$T(K)$ & $V\left(\mu s^{-1}\right)$ \\
\hline 100 & $0.022 \pm 0.006$ \\
110 & $0.084 \pm 0.016$ \\
120 & $0.125 \pm 0.035$ \\
140 & $0.402 \pm 0.023$ \\
160 & $0.699 \pm 0.036$ \\
\hline
\end{tabular}


Table II

Transverse-field widths and asymmetries

\begin{tabular}{|c|c|c|c|c|c|c|}
\hline Run & Sample & $\mathrm{H}(\mathrm{Oe})$ & $\mathbf{T}(\mathbf{K})$ & Hist. & $\mathbf{A}$ & $\sigma\left(\mu \mathrm{s}^{-1}\right)$ \\
\hline 115 & LBL & 100 & $2.65 \pm 0.05$ & $\begin{array}{l}\mathrm{L} \\
\mathrm{R} \\
\mathrm{F} \\
\mathrm{B} \\
\end{array}$ & $\begin{array}{l}.2425(20) \\
.2543(20) \\
.2471(34) \\
.1966(16) \\
\end{array}$ & $\begin{array}{l}.3144(58) \\
.3106(58) \\
.3002(101) \\
.3043(60) \\
\end{array}$ \\
\hline 121 & LBL & 100 & $4.2 \pm 0.1$ & $\begin{array}{l}\text { L } \\
\text { R } \\
\text { F } \\
\text { B }\end{array}$ & $\begin{array}{l}.2451(15) \\
.2542(14) \\
.2552(26) \\
.1976(6)\end{array}$ & $\begin{array}{l}.3410(45) \\
.3359(43) \\
.3413(78) \\
.3388(45) \\
\end{array}$ \\
\hline 126 & LBL & 100 & $0.74 \pm 0.01$ & $\begin{array}{l}\text { L } \\
\text { R } \\
\text { F } \\
\text { B }\end{array}$ & $\begin{array}{l}.2421(16) \\
.2545(16) \\
.2493(28) \\
.1935(13)\end{array}$ & $\begin{array}{l}.2786(46) \\
.2899(45) \\
.2833(81) \\
.2828(48)\end{array}$ \\
\hline 128 & CERN & 100 & $4.2 \pm 0.02$ & $\begin{array}{l}\text { L } \\
\text { R } \\
\text { F } \\
\text { B }\end{array}$ & $\begin{array}{l}.2414(16) \\
.2425(16) \\
.2513(27) \\
.1978(13)\end{array}$ & $\begin{array}{r}.3311(47) \\
.3441(51) \\
.3296(79) \\
.3394(49) \\
\end{array}$ \\
\hline 131 & CERN & 100 & $0.49 \pm 0.01$ & $\begin{array}{l}\mathbf{L} \\
\mathbf{R} \\
\mathbf{F} \\
\mathrm{B}\end{array}$ & $\begin{array}{r}.2386(14) \\
.2406(15) \\
.2523(24) \\
.1936(12) \\
\end{array}$ & $\begin{array}{r}.2439(40) \\
.2536(43) \\
.2544(67) \\
.2465(44) \\
\end{array}$ \\
\hline 135 & CERN & 100 & $1.1 \pm 0.05$ & $\begin{array}{l}\mathrm{L} \\
\mathrm{R} \\
\mathrm{E} \\
\mathrm{B}\end{array}$ & $\begin{array}{l}.2403(16) \\
.2444(17) \\
.2552(27) \\
.1935(14) \\
\end{array}$ & $\begin{array}{l}.2757(47) \\
.2824(49) \\
.2873(77) \\
.2914(53) \\
\end{array}$ \\
\hline 812 & LBL & 86 & $4.8 \pm 0.1$ & $\begin{array}{l}\text { L } \\
\mathrm{R} \\
\mathrm{F} \\
\mathrm{B}\end{array}$ & $\begin{array}{l}.2709(13) \\
.2689(11) \\
.2686(22) \\
.2104(14) \\
\end{array}$ & $\begin{array}{r}.3280(34) \\
.3202(31) \\
.3270(70) \\
.3252(50) \\
\end{array}$ \\
\hline 815 & $\overline{\mathrm{LBL}}$ & 40 & $4.8 \pm 0.1$ & $\begin{array}{l}\mathbf{L} \\
\mathbf{R} \\
\text { E } \\
\text { B }\end{array}$ & $\begin{array}{l}.2669(22) \\
.2668(19) \\
.2713(38) \\
.2095(23) \\
\end{array}$ & $\begin{array}{l}\cdot 3259(57) \\
.3198(51) \\
.3324(114) \\
.3437(84)\end{array}$ \\
\hline 818 & LBL & 80 & $2.43 \pm 0.01$ & $\begin{array}{l}\mathrm{L} \\
\mathrm{R} \\
\mathrm{E} \\
\mathrm{B}\end{array}$ & $\begin{array}{l}.2691(19) \\
.2676(17) \\
.2673(35) \\
.2118(21) \\
\end{array}$ & $\begin{array}{l}.3032(52) \\
.3123(48) \\
.3166(108) \\
.3104(73) \\
\end{array}$ \\
\hline 819 & LBL & 80 & $0.61 \pm 0.01$ & $\begin{array}{l}\text { L } \\
\text { R } \\
\text { F } \\
\text { B }\end{array}$ & $\begin{array}{l}.2656(17) \\
.2650(16) \\
.2648(31) \\
.2048(18)\end{array}$ & $\begin{array}{l}.2598(46) \\
.2617(42) \\
.2659(98) \\
.2567(65)\end{array}$ \\
\hline
\end{tabular}


Table III

Zero-field Dipolar Widch

\begin{tabular}{|c|c|c|c|c|}
\hline Run & Sample & $T(K)$ & Hists. & $\Delta_{z}\left(\mu s^{-1}\right)$ \\
\hline \multirow[b]{2}{*}{114} & \multirow{3}{*}{ LBL } & \multirow{3}{*}{$0.46 \pm 0.002$} & $\bar{F}$ & $.397(14)$ \\
\hline & & & & \\
\hline \multirow{3}{*}{116} & & & $\frac{B}{F}$ & $\frac{.390(10)}{.392(10)}$ \\
\hline & \multirow[t]{2}{*}{ LBL } & \multirow{2}{*}{$2.72 \pm 0.03$} & & \\
\hline & & & B & $.395(5)$ \\
\hline \multirow{2}{*}{117} & \multirow[b]{2}{*}{ LBL } & \multirow{2}{*}{$0.50 \pm 0.007$} & $F$ & $.401(13)$ \\
\hline & & & B & $.397(12)$ \\
\hline \multirow[b]{2}{*}{119} & \multirow[b]{2}{*}{ LBL } & \multirow[b]{2}{*}{$4.20 \pm 0.05$} & $\mathrm{~F}$ & $.361(11)$ \\
\hline & & & B & $.382(5)$ \\
\hline \multirow[b]{2}{*}{125} & \multirow[b]{2}{*}{ LBL } & \multirow{2}{*}{$0.73 \pm 0.01$} & $\mathbf{F}$ & $.380(12)$ \\
\hline & & & B & $.389(8)$ \\
\hline 129 & CERN & $4.3 \pm 0.1$ & B & $.359(5)$ \\
\hline \multirow[b]{2}{*}{132} & \multirow[b]{2}{*}{ CERN } & \multirow[b]{2}{*}{$0.5 \underline{0+0.01}$} & $\mathbf{F}$ & $.381(11)$ \\
\hline & & & B & $.393(10)$ \\
\hline \multirow{3}{*}{134} & \multirow{3}{*}{ CERN } & \multirow{3}{*}{$1.10 \underline{0.01}$} & $\bar{F}$ & $.400(11)$ \\
\hline & & & & \\
\hline & & & B & $-389(8)$ \\
\hline \multirow{2}{*}{816} & \multirow{2}{*}{ LBL } & \multirow{2}{*}{$5.15 \pm 0.05$} & $\mathbf{F}$ & $.397(7)$ \\
\hline & & & $\mathrm{b}$ & $.399(5)$ \\
\hline \multirow[b]{2}{*}{817} & \multirow[b]{2}{*}{ LBL } & \multirow[b]{2}{*}{$2.35 \pm 0.03$} & $\mathbf{F}$ & $.381(8)$ \\
\hline & & & $B$ & $.385(5)$ \\
\hline 820 & \multirow[b]{2}{*}{ LBL } & \multirow[b]{2}{*}{$0.63+0.01$} & $\mathbf{F}$ & $.399(7)$ \\
\hline $\begin{array}{l}+822 \\
+824\end{array}$ & & & B & $.369(5)$ \\
\hline
\end{tabular}


Table IV

Zero-fleld Hopping Rates

\begin{tabular}{|c|c|c|c|c|c|c|c|}
\hline Run & Sample & $T(K)$ & Hist. & $V\left(\mu_{\mathrm{s}}^{-1}\right)$ & terror & -error & $x^{2} / \mathrm{NDF}$ \\
\hline \multirow{2}{*}{114} & \multirow{2}{*}{ LBL } & \multirow{2}{*}{$0.46 \pm 0.002$} & F & .3349 & 70 & 214 & $179 / 144$ \\
\hline & & & B & $.26,76$ & 91 & 165 & $197 / 145$ \\
\hline \multirow[b]{2}{*}{116} & \multirow[b]{2}{*}{ LBL } & \multirow{2}{*}{$2.72 \pm 0.03$} & F & .1385 & 177 & 165 & $161 / 144$ \\
\hline & & & B & .0990 & 96 & 96 & $169 / 145$ \\
\hline \multirow{3}{*}{117} & \multirow{3}{*}{ LBL } & \multirow{3}{*}{$0.50 \pm 0.007$} & F & .2942 & 396 & 361 & $178 / 144$ \\
\hline & & & & & & & \\
\hline & & & B & .3346 & 121 & 199 & $170 / 145$ \\
\hline \multirow{2}{*}{119} & \multirow{2}{*}{ LBL } & \multirow{2}{*}{$4.20 \pm 0.05$} & $\bar{F}$ & .2418 & 113 & 145 & $155 / 144$ \\
\hline & & & B & .2031 & 175 & 138 & $147 / 145$ \\
\hline \multirow[b]{2}{*}{125} & \multirow[b]{2}{*}{ LBL } & \multirow[b]{2}{*}{$0.73 \pm 0.01$} & $F$ & .4417 & 390 & 230 & $130 / 144$ \\
\hline & & & B & .5078 & 223 & 225 & $155 / 145$ \\
\hline 129 & CERN & $4.3+0.1$ & $\mathrm{~B}$ & .1707 & 103 & 106 & $181 / 145$ \\
\hline \multirow[b]{2}{*}{132} & \multirow[b]{2}{*}{ CERN } & \multirow[b]{2}{*}{$0.50 \pm 0.01$} & $\vec{F}$ & .4070 & 169 & 299 & $137 / 144$ \\
\hline & & & B & .4135 & 171 & 168 & $157 / 145$ \\
\hline \multirow[b]{2}{*}{134} & \multirow[b]{2}{*}{ CERN } & \multirow[b]{2}{*}{$1.10 \pm 0.01$} & $F$ & .3594 & 206 & 214 & $125 / 144$ \\
\hline & & & B & .3610 & 143 & 137 & $152 / 145$ \\
\hline \multirow[b]{2}{*}{816} & \multirow[b]{2}{*}{ LBL } & \multirow[b]{2}{*}{$5.15 \pm 0.05$} & F & 0.0000 & 31 & - & $185 / 141$ \\
\hline & & & B & .0595 & - & 290 & $181 / 141$ \\
\hline \multirow{2}{*}{817} & \multirow{2}{*}{ LBL } & \multirow{2}{*}{$2.35 \pm 0.03$} & $\bar{F}$ & .2264 & - & 106 & $193 / 141$ \\
\hline & & & $\mathrm{B}$ & .2675 & 138 & 109 & $145 / 141$ \\
\hline \multirow{2}{*}{$\begin{array}{r}820 \\
+822 \\
+824 \\
\end{array}$} & \multirow{2}{*}{ LBL } & \multirow{2}{*}{$0.63 \pm 0.01$} & $F$ & .3577 & 129 & 170 & $229 / 141$ \\
\hline & & & B & .4873 & 186 & 139 & $149 / 141$ \\
\hline
\end{tabular}


Table V

Zero- and Transverse-fleld Hopping Rates

$T(k) \quad v_{z f}\left(\mu s^{-1}\right) \quad v_{t}\left(\mu s^{-1}\right)$

$4.8 \pm 0.1 \quad \overline{6} 409 \pm 0.031$

$5.14 \pm 0.05 \quad 0.092 \pm 0.012$

$2.43 \pm 0.01$

$2.35 \pm 0.03$

$0.261 \pm 0.014$

$0.509 \pm 0.015$

$0.61 \pm 0.01$

$0.63 \pm 0.01 \quad 0.489 \pm 0.034$

$0.822 \pm 0.042$ 
Flgure captions

(1) Transverse-field relaxation rate vs. temperature in folycrystaline copper. The rate $\Lambda(T)$ is defined in a shape-1ndependtnt manner as the inverse of the time taken for the polarization to decay to $1 / e$ of 1 ts inftial value. a) $\wedge$ vs. $T$. b) Arrhentus plot of correlation times obtalned from 1/o times of Eq. III.11. (From Ref. 5.)

(2) Potential energy for hydrogenic interstitials in a rigld ou lattice. The ground state energies of the proton and the $\mu^{+}$at the octahedral (0) site are Indicated. The well at the tetrahedral (T) site is too shallow for a $\mu^{+}$bound state to exist. (From Ref. 19.)

(3) Range curve for surface $\mu^{+}$in mylar. Thts represents the fraction of the $\mu^{+}$beam surviving after traversing a given amount of mater1a1. (From Ref. 2.)

(4) Plan view of the "Eagle" $\mu^{+} \mathrm{SR}$ spectrometer vacuum chamber with cryostat.

(5) Data acqingition electronics. Not shown are scaler connections and delay cables necessary for pulse timing.

(6) Tining diagrams for typical esents. T represtncs the adfustable data gate length. 
(7) Schematic view of ${ }^{3}$ He refrigerator installed in the tail of the ${ }^{4}$ Be gas-flow cryostat. Not shown 1 s the $77 \mathrm{~K}$ radiation shield with a $0.0003^{\prime \prime}$ aluminized Mylar window.

(8) a) Example of a "raw" transverse-field $\mu^{+}$Sk spectrum, taken In copper at 83 oe and $4.2 \mathrm{R}$. b) The same data reduced to show the asymmetry.

(9) a) zero-field and b) transverse-ffeld relaxation functions wh th $\Delta_{z}^{2}=5 / 2 \Delta_{x}^{2}$. In both cases the time axis is $\Delta_{z} t$. The curves 1n a) are from the theory of Ref. 4, and those In b) are from Eq . III. 11.

(10) Stat1c transverse dipolar width vs. magnetic field. (From Ref. 7.)

(11) Arrhenius plot of hopping rate in copper derived from zero-field relaxation near $100 \mathrm{k}$.

(12) Transverse-field line width in copper at low temperature. Our data are superimposed on that of Hartmann, et. al. (Ref, 23). For our data, the errors are smaller than the point sizes.

Ref. 23 -- Open squares: Cu polycrystal, 520 0e Open circles: Al polycrystal, 120 De Filled c1rcles: Al polycrystal, $5200 \mathrm{oe}$

This work -- F1lled triangles: Cu crystal, 80 Oe Open triangles: Cu polycrystal, 80 0e Crossed clrcle: Al Polycrystal, 80 Oe 
(13) Zero-field width vs. temperature, from teighted averages of forward and backward spectra. FH1led polnts are LBL copper crystal, open points are CERN cof er used tn Ref. 23.

(14) Hopping rate from zero-field relaxation at low temperature. Filled points are from the LBL copper, and open points are from the CERN copper used in Ref. 23.

(15) Backward asymmetry in zero fleld at several temperatures.

(16) Zero-field and transverse-field hopping rates in copper, from simultaneous fits to forward and backward spectra*

(17) Schematic plot of transverse-fleld width vs. temperature for trap-1imited diffusion. The dotted line indicates the effect of Increasing the trap concentration, thereby reducing $T_{t}$.

(18) Zero-field relaxation functions for trap-limited diffusion. (From Ref. 71.) 


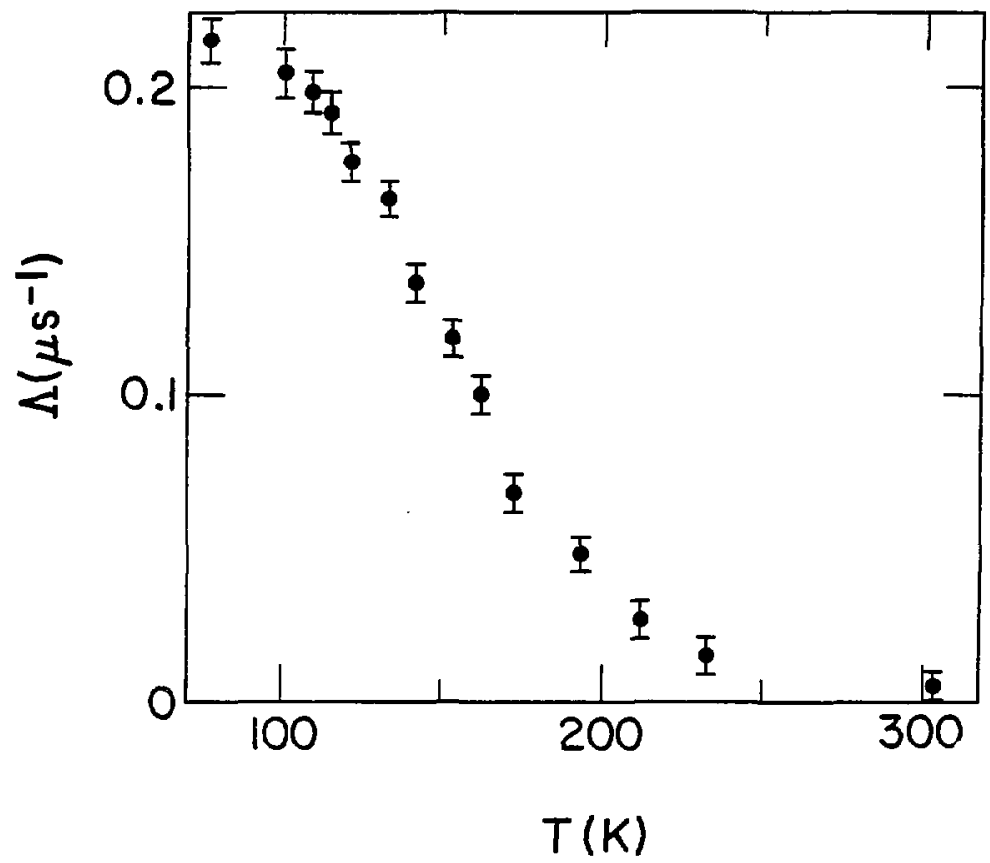

XBL 824-537

Fig. la 


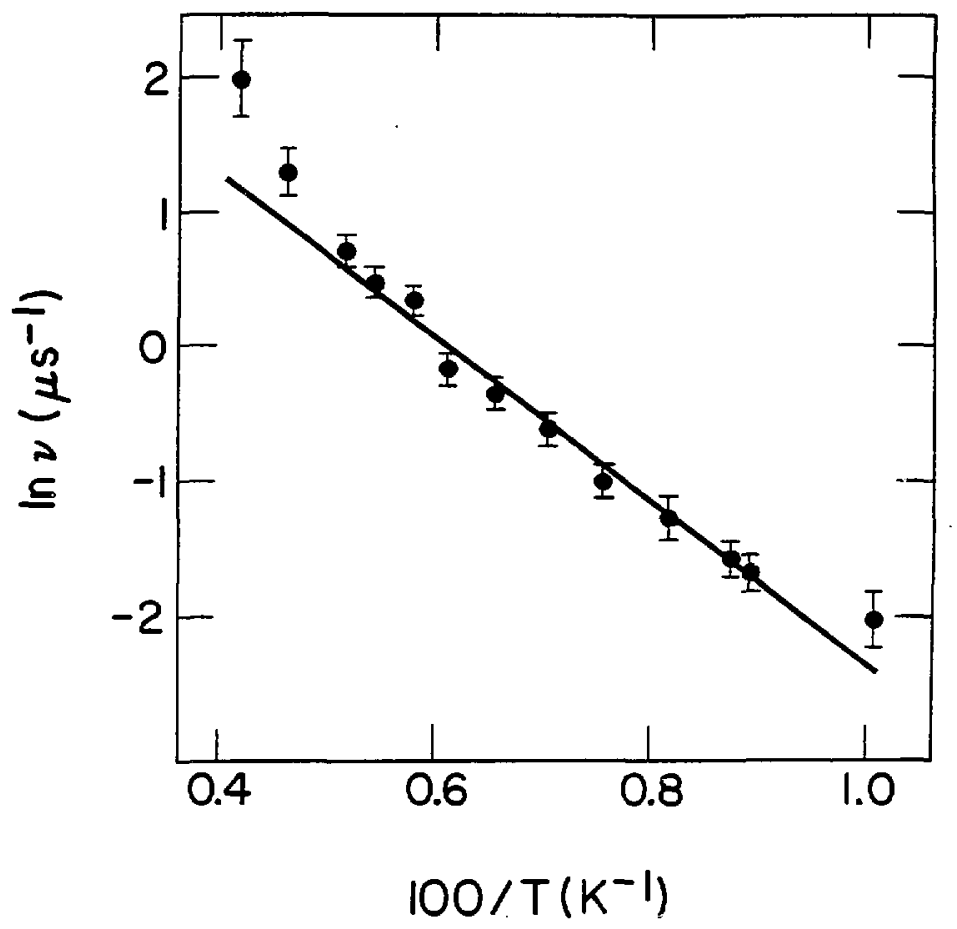

XBL 824-536

Fig. Ib 


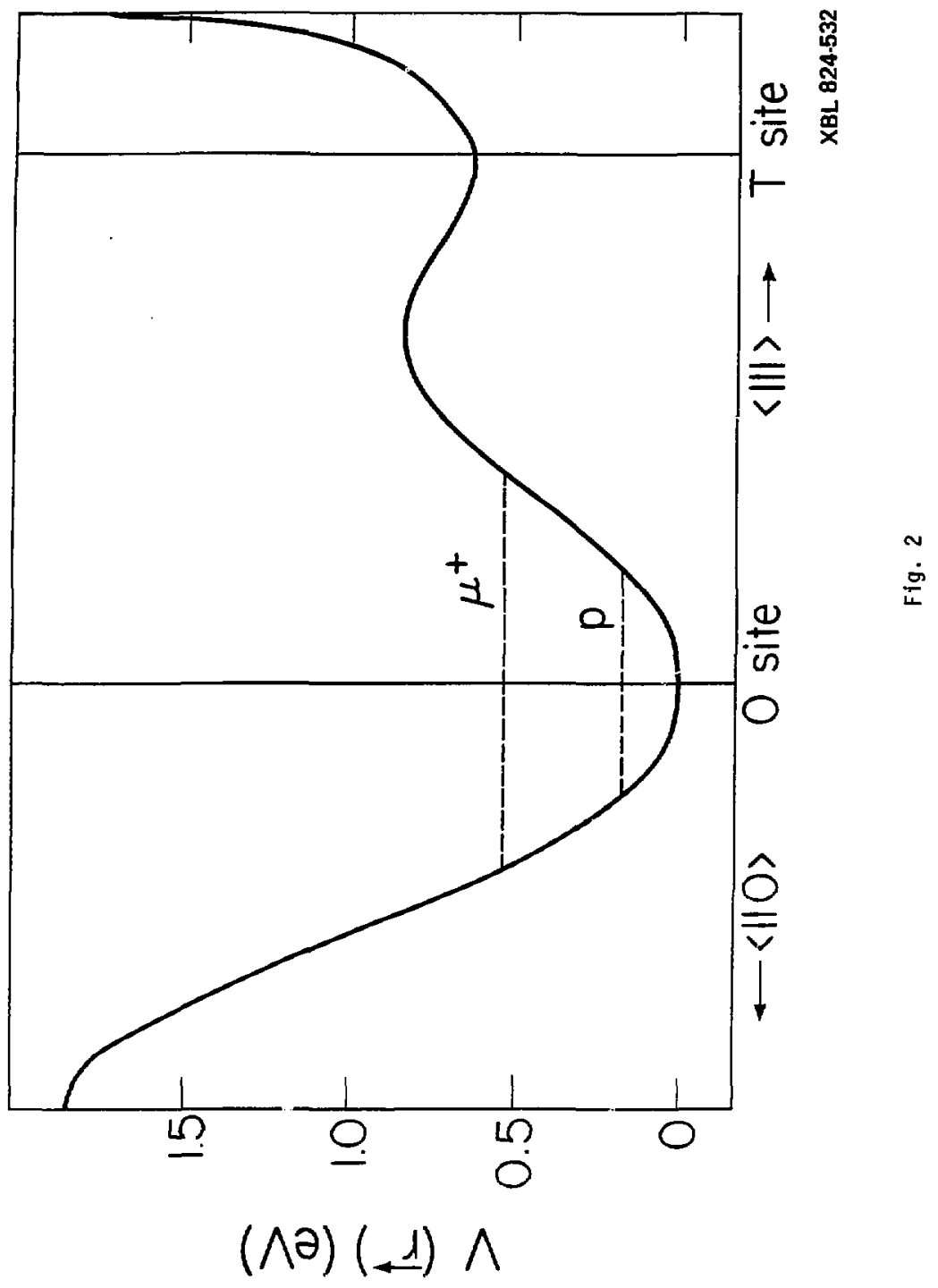




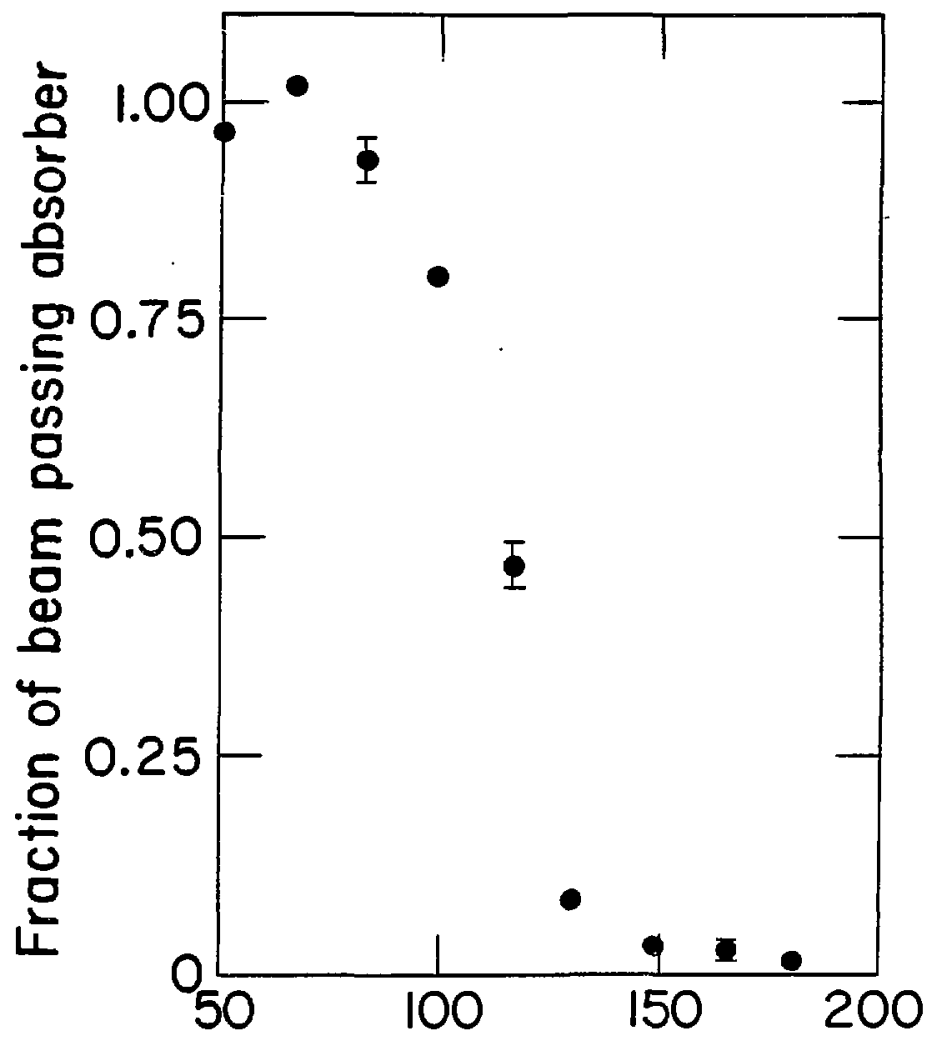

Absorber thickness $\left(\mathrm{mg} / \mathrm{cm}^{2}\right)$

XBL 324-535

Fig. 3 
77K Heat Shield

(1/4 Mil Aluminized Mylar)

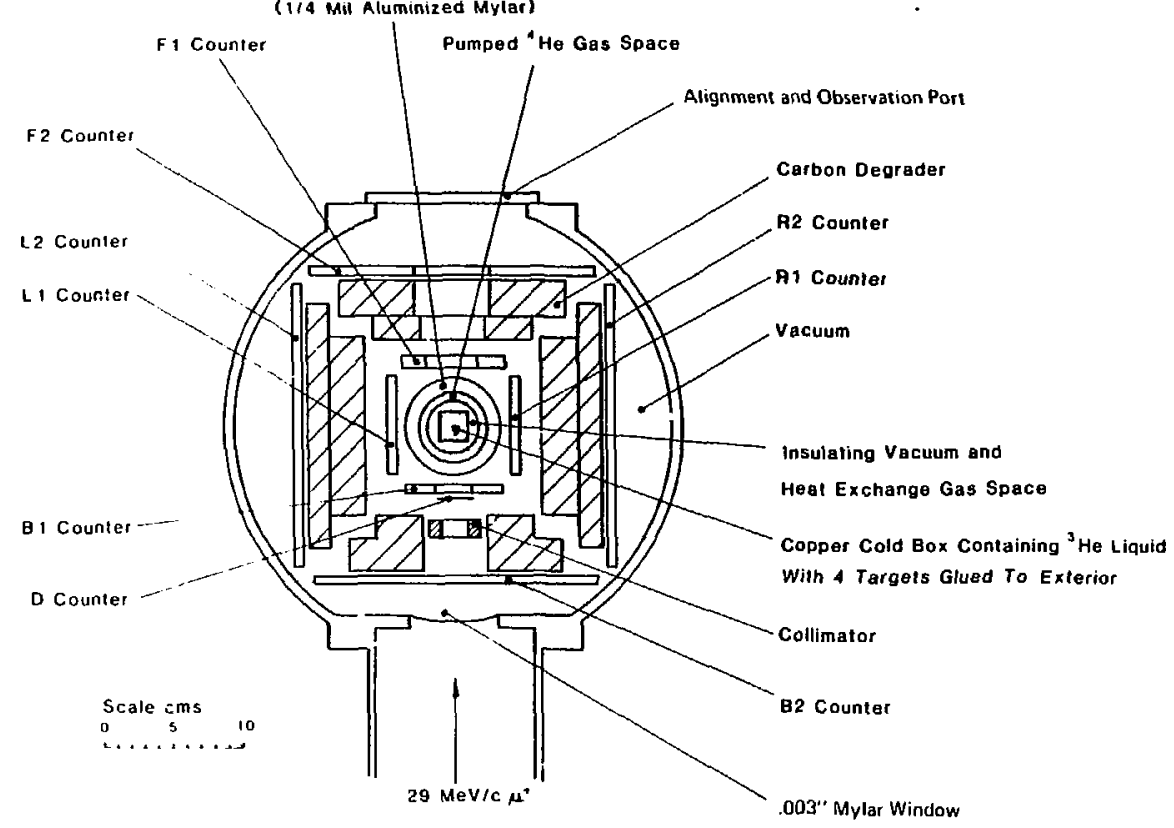

XEL $817-10875$

Fig. 4 


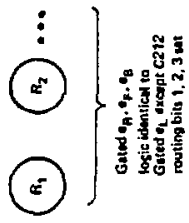

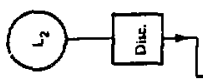
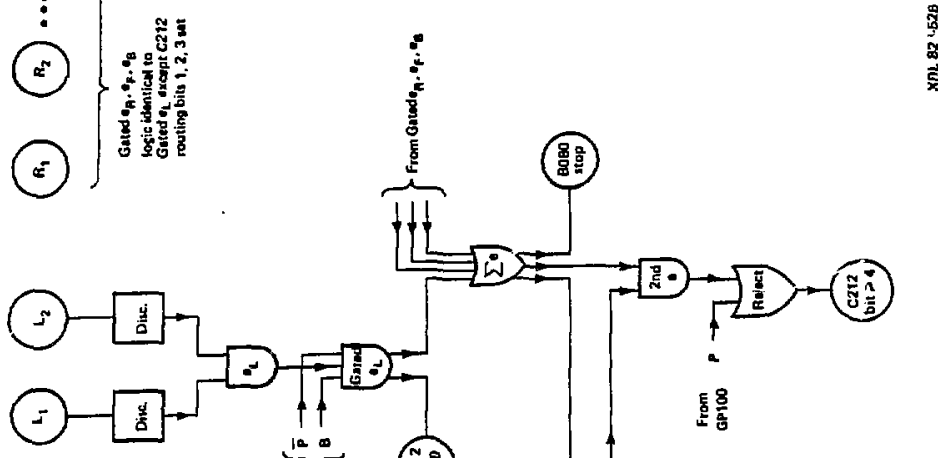

影 送望

(3i⿱ 욜

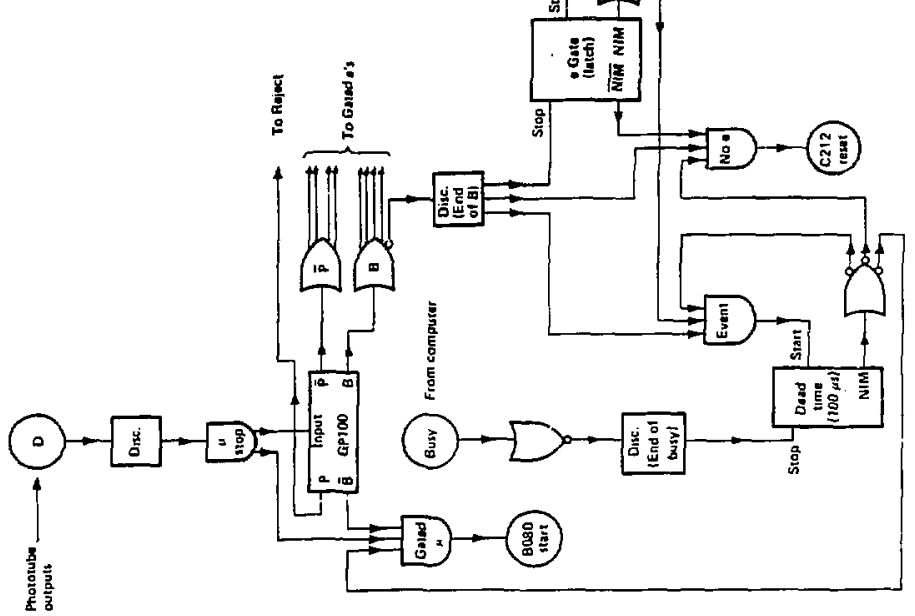


Good Event

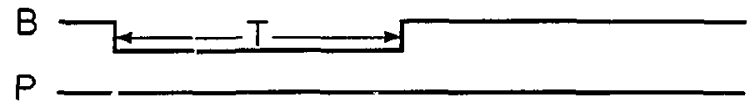

e Gate

Routing

Bits

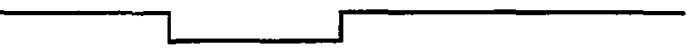

Reject

Dead

Time

Computer

Busy

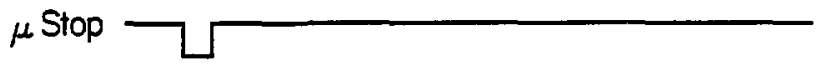

Goted e
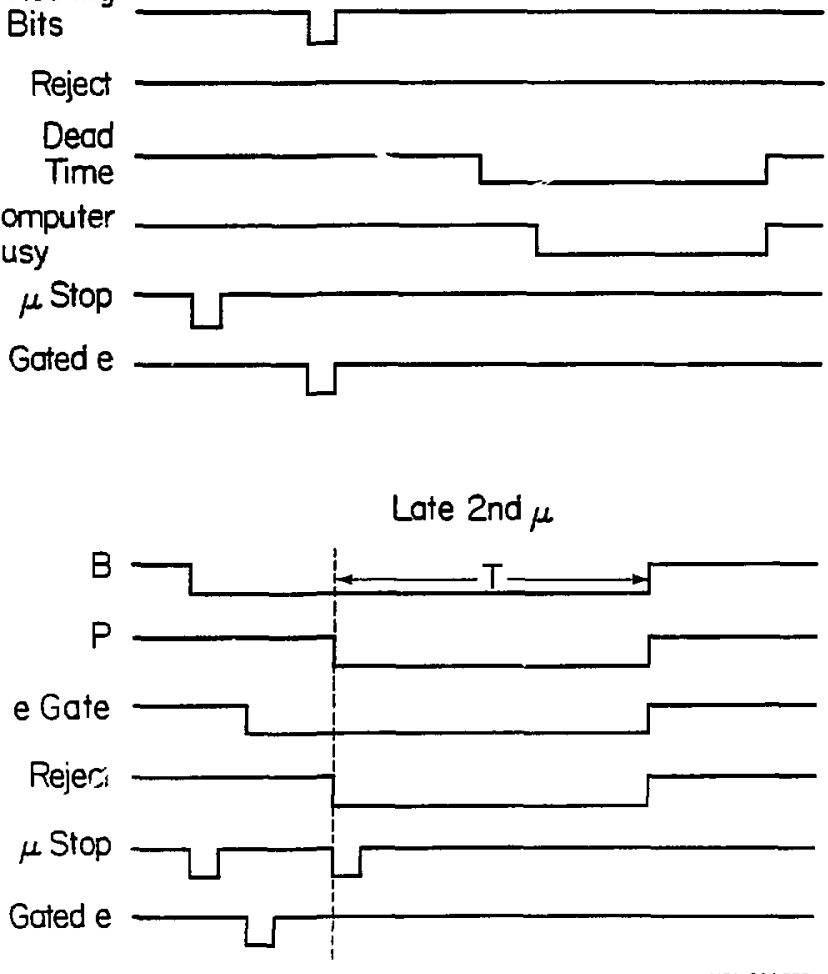

XBL 824.528

Fig. 6

(cuntinued) 

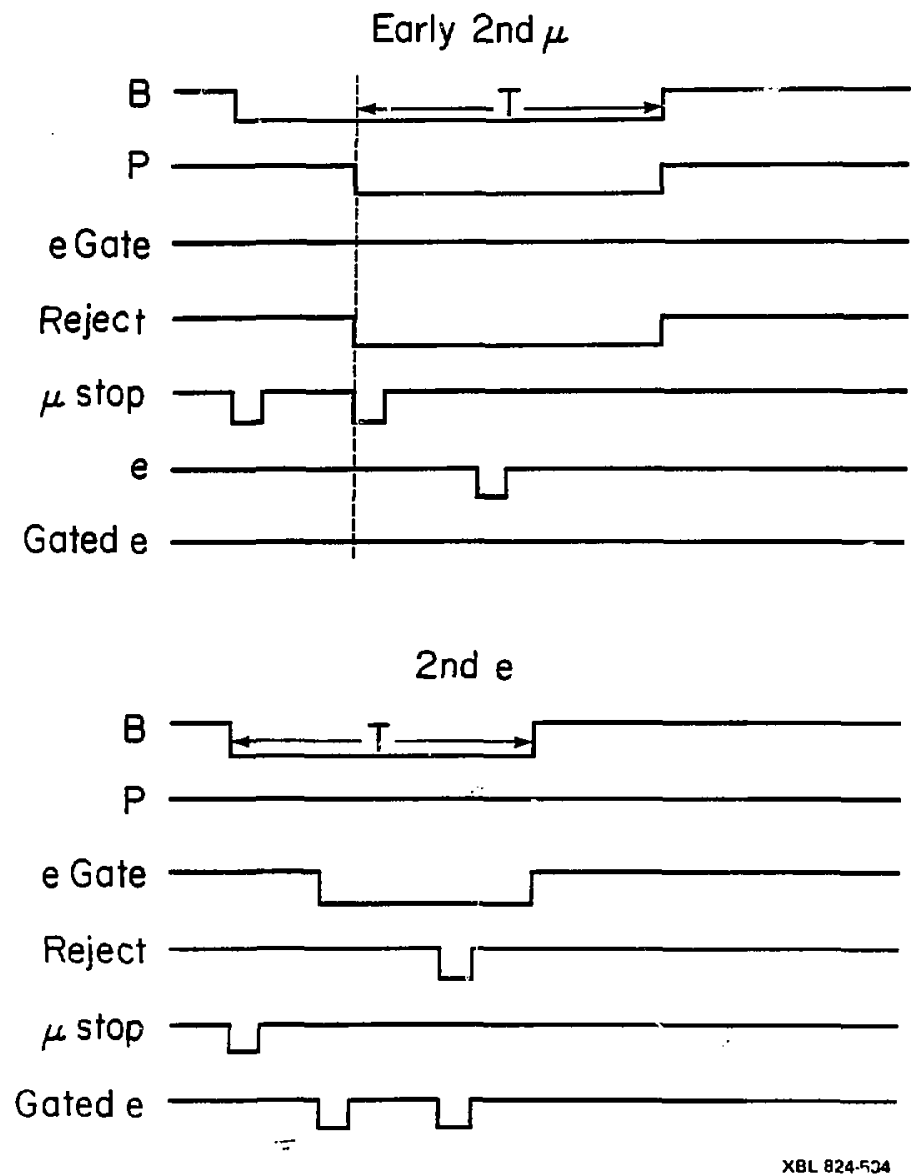

Fig. 6 


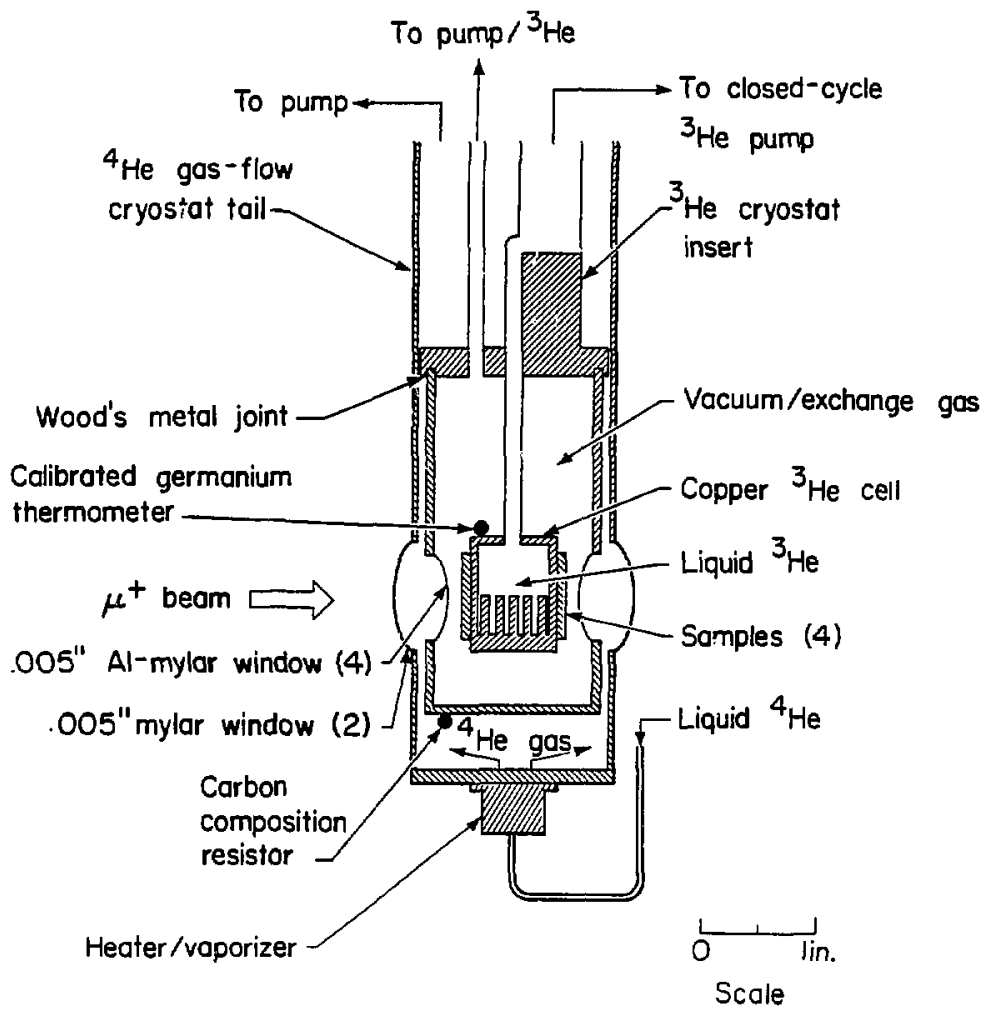

XBL 824.531

Fig. $?$ 


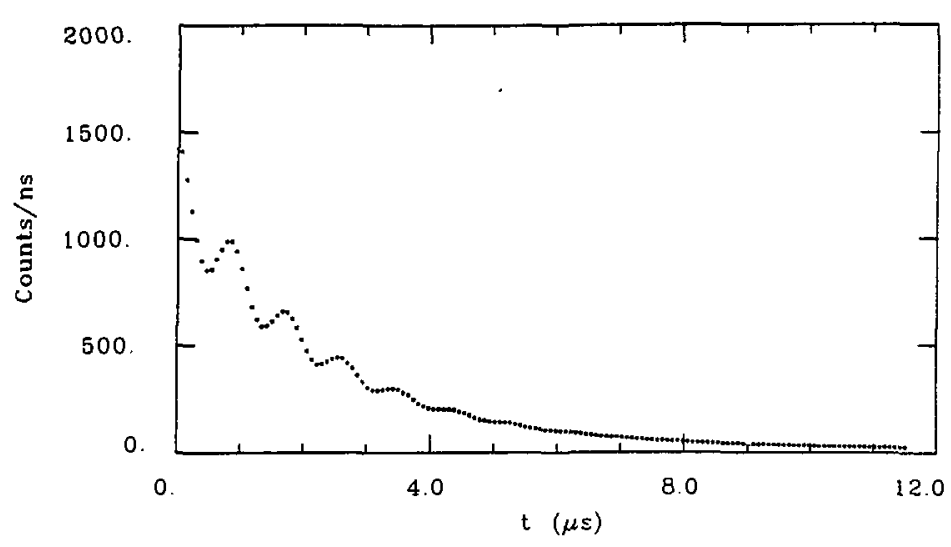

(a)

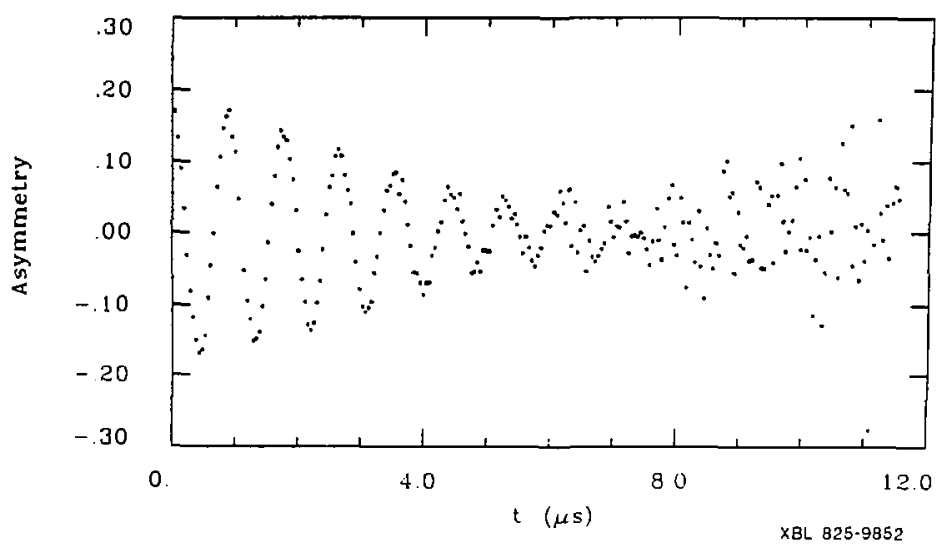

(b)

Fig. 8 


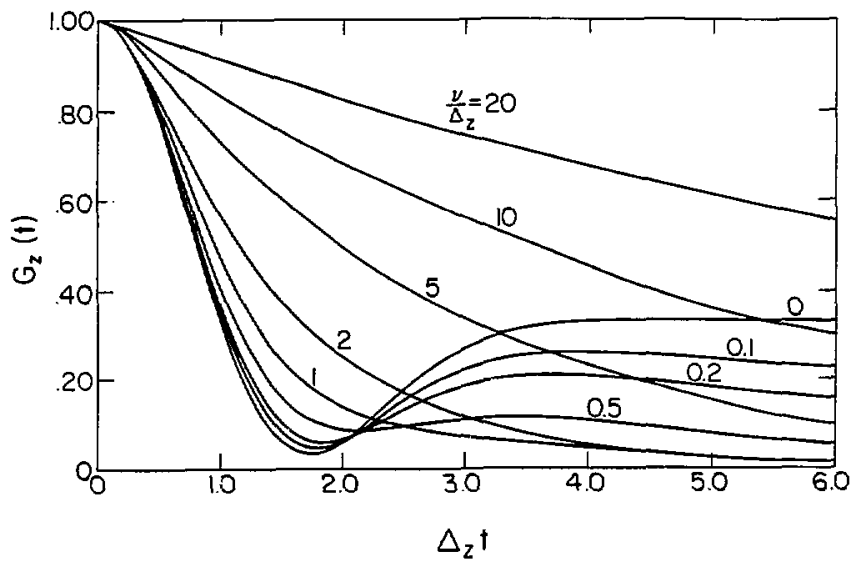

(a)

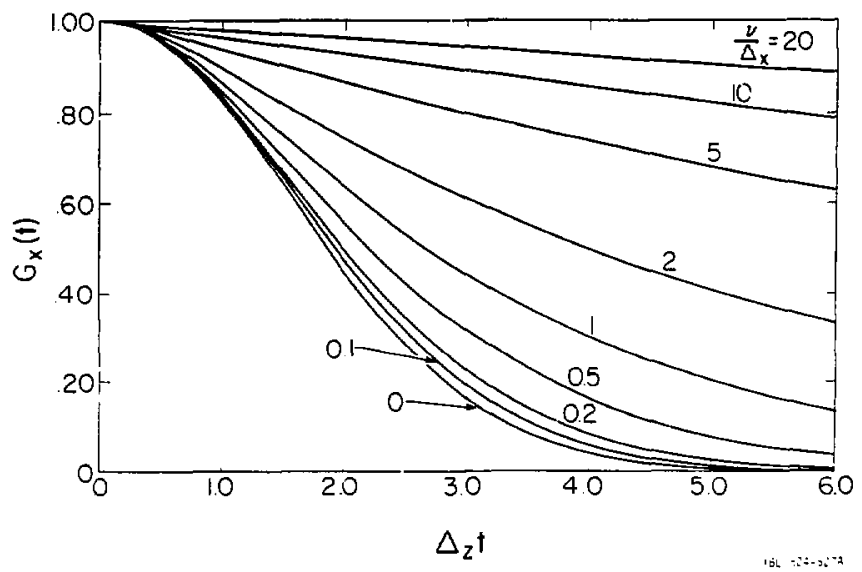

(b)

Fig. 9 


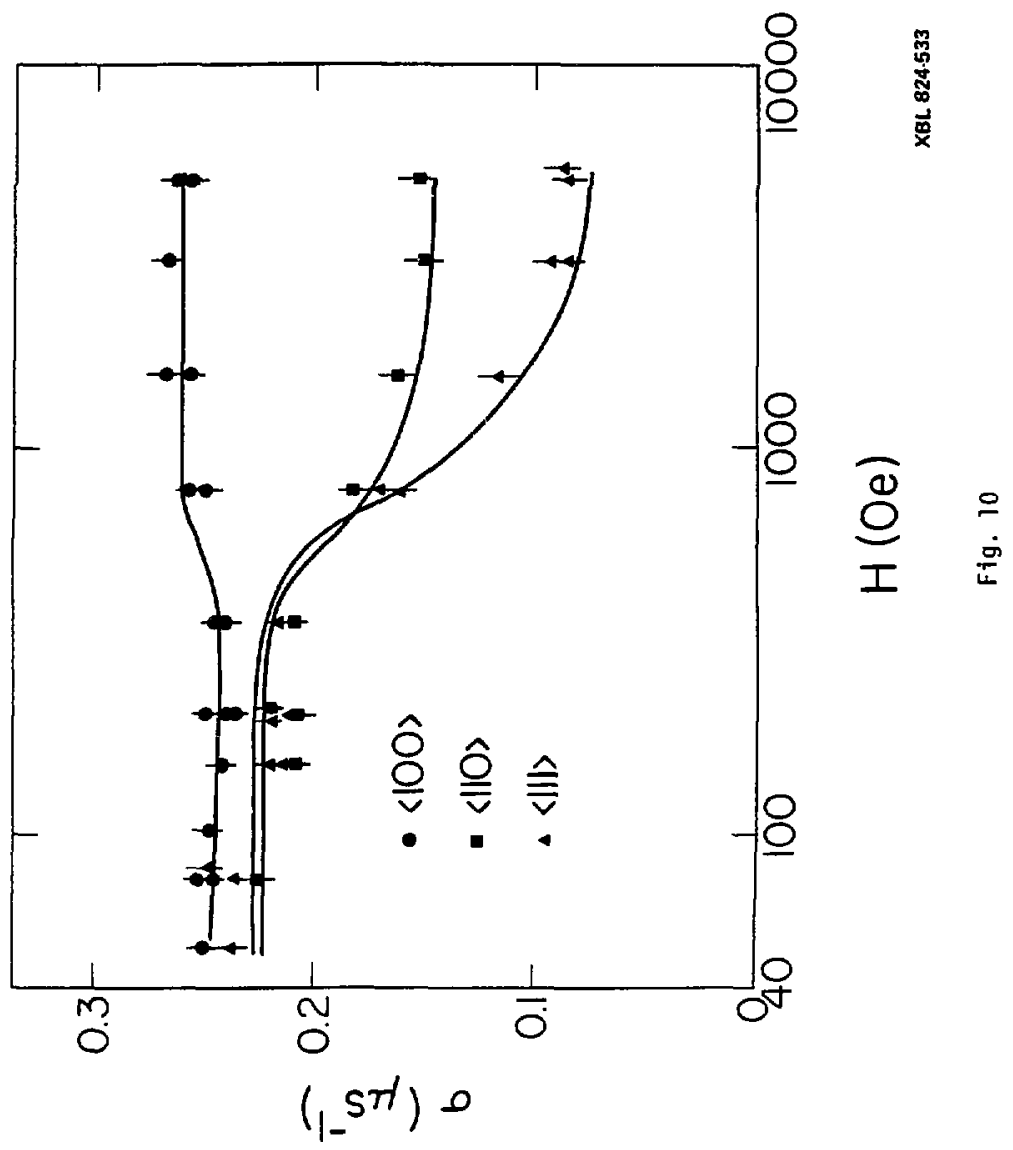




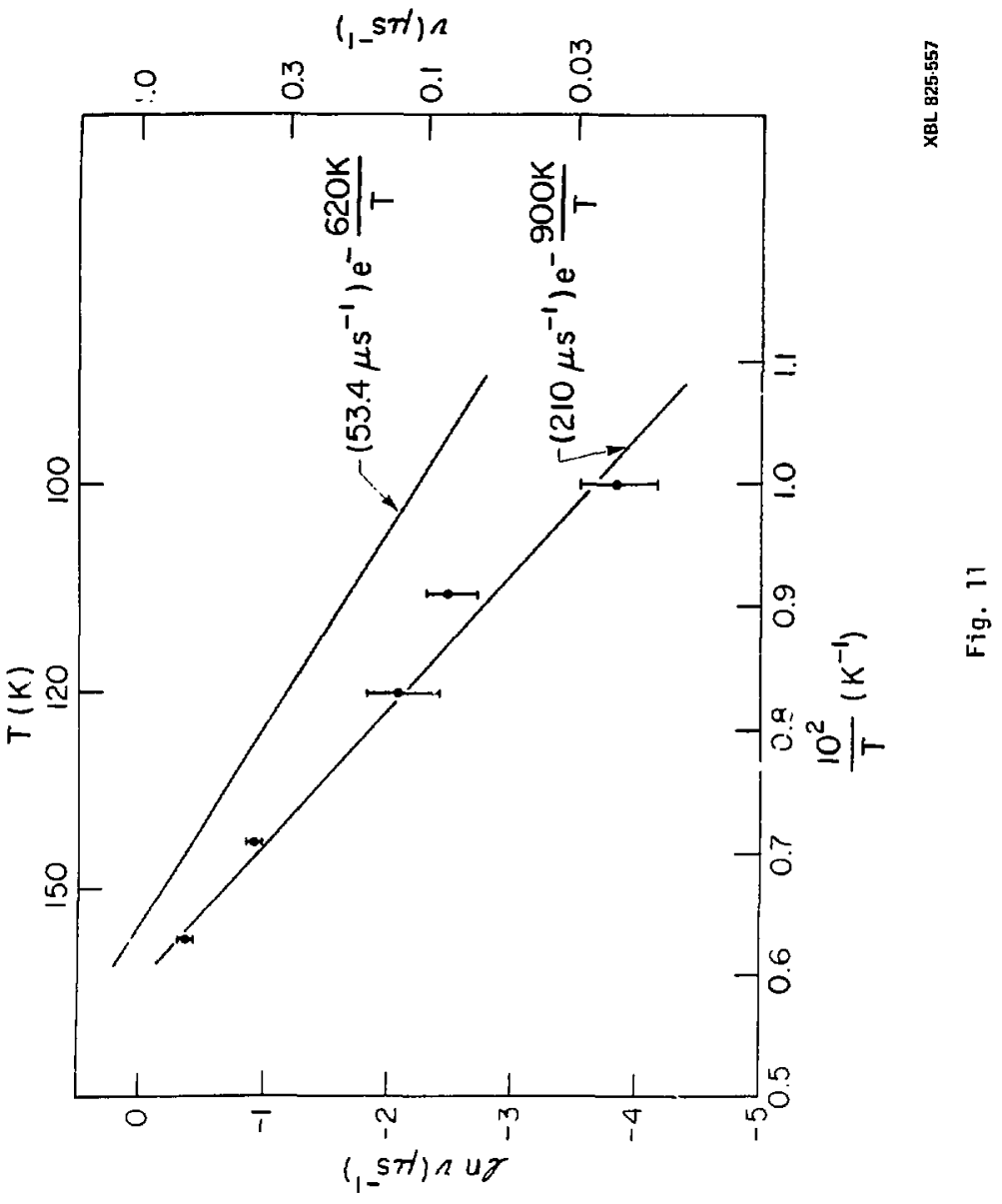


$9 \angle 80 T-\angle$ โ8 $78 X$

(y) 1

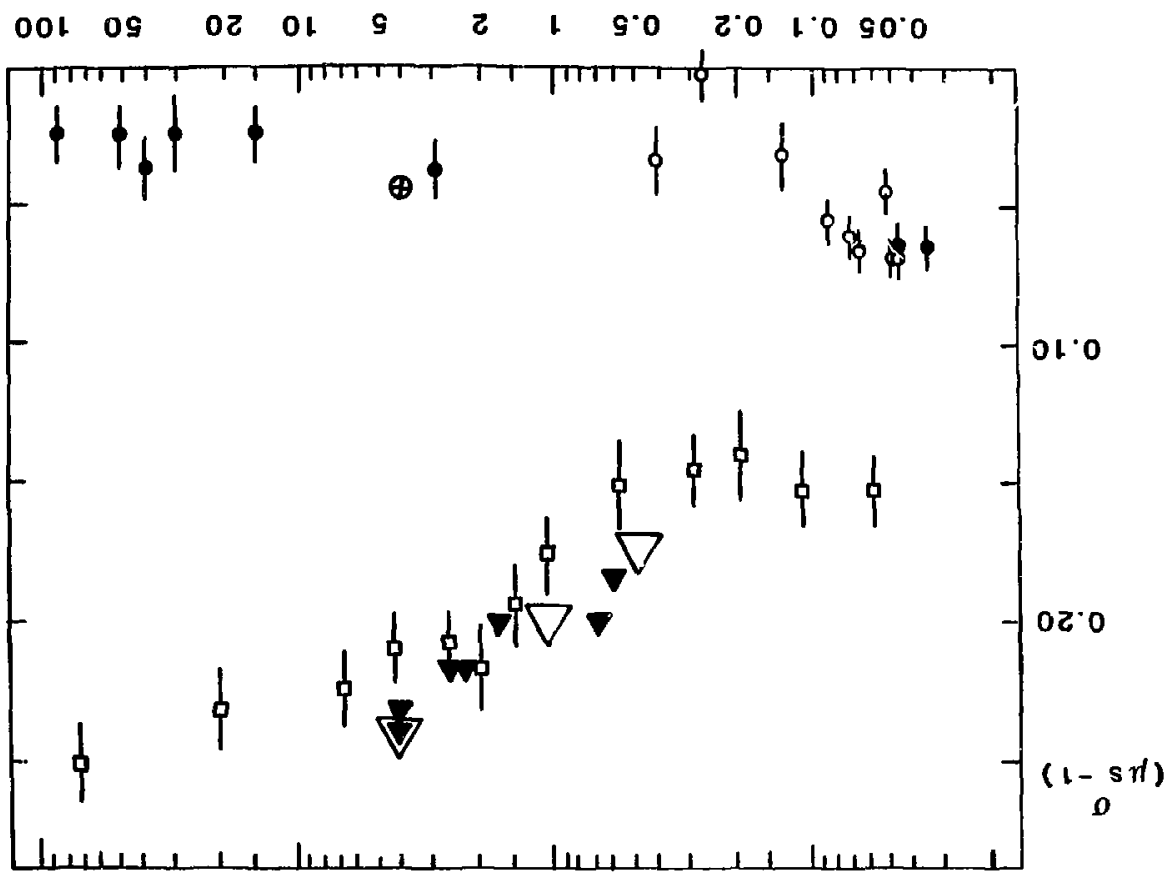




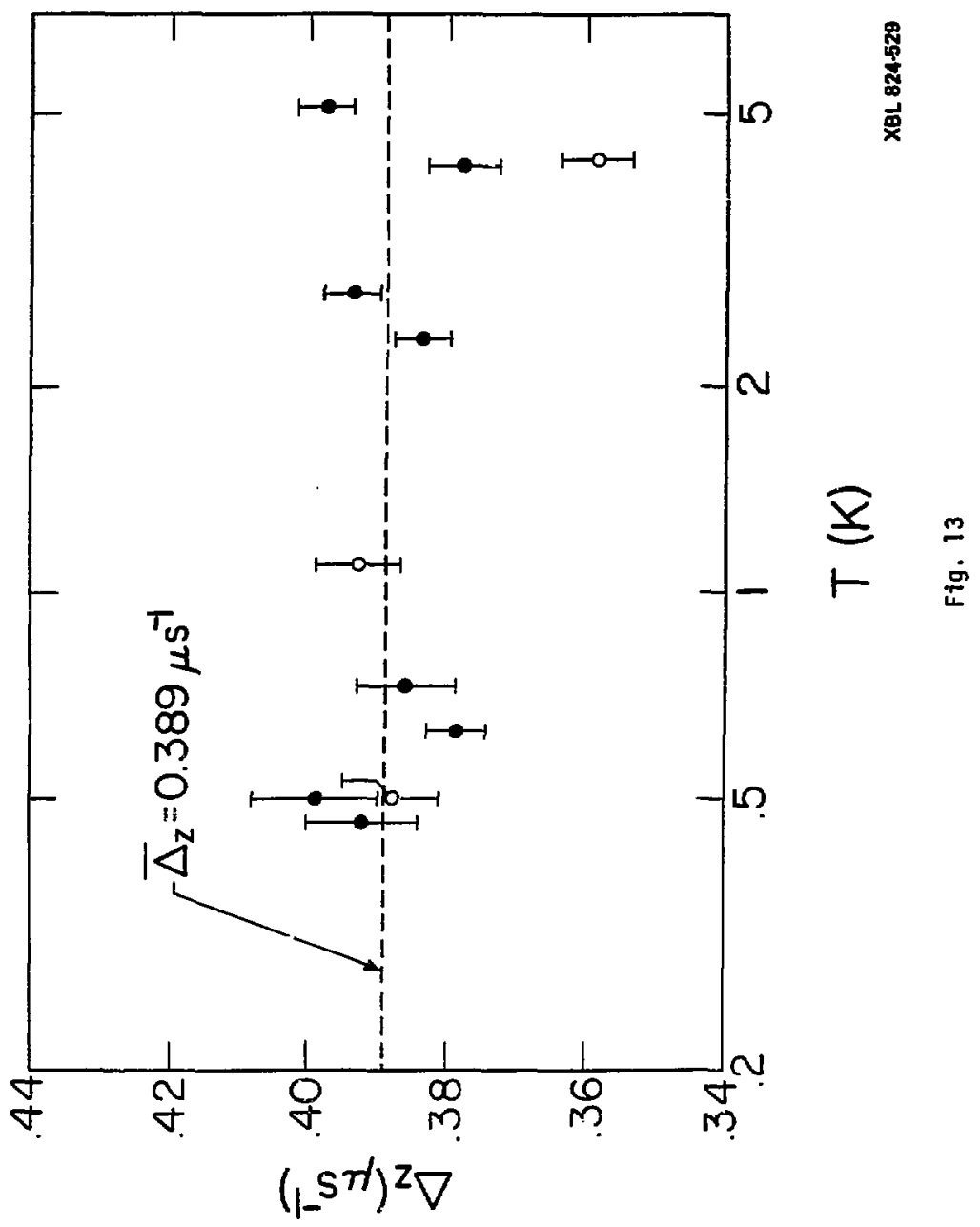




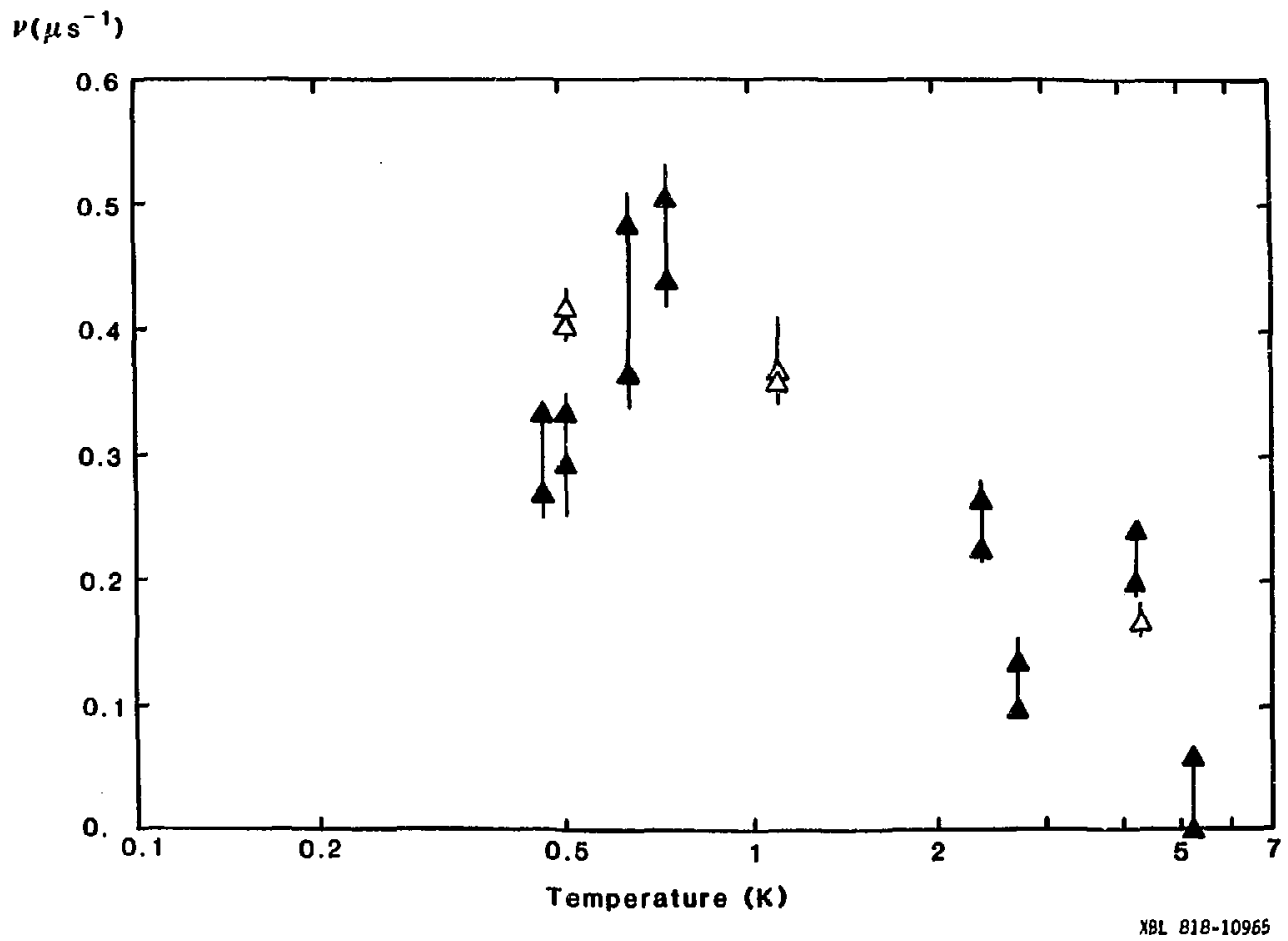

Fig. 14 


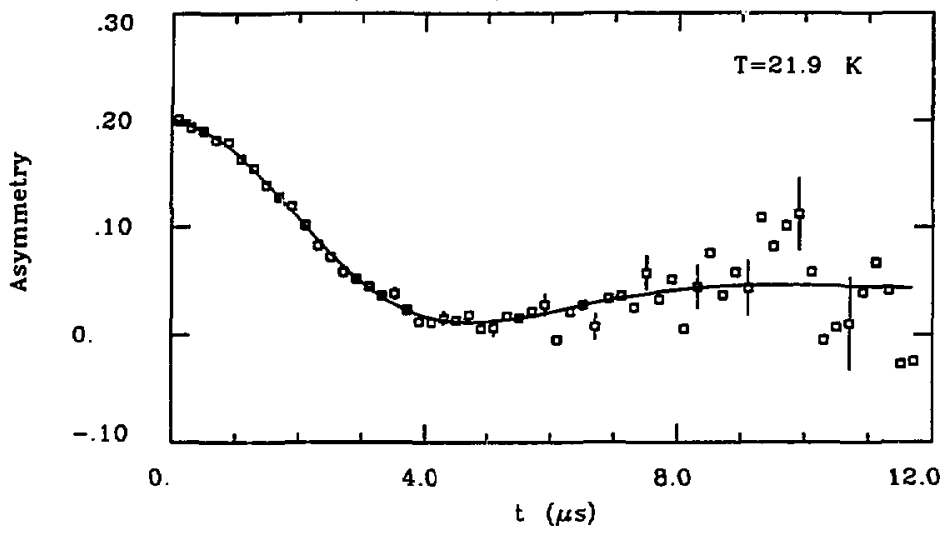

(a)

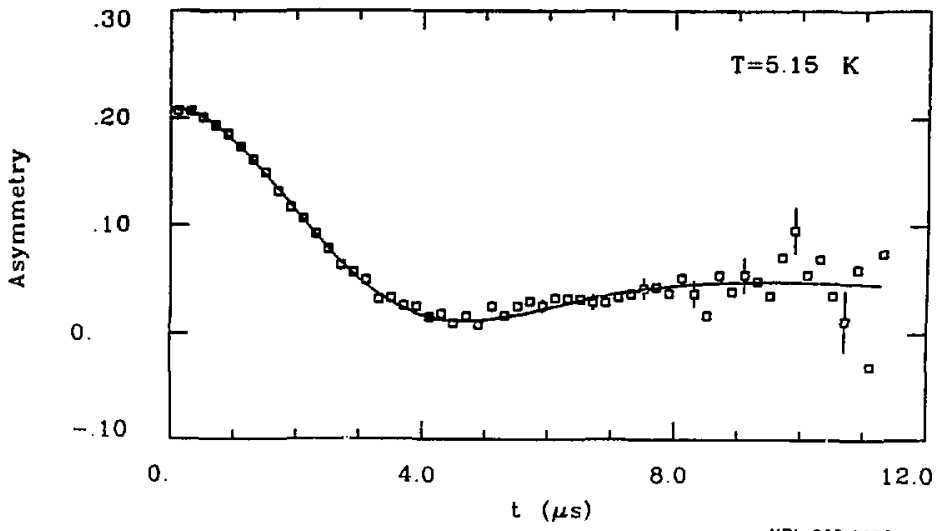

(b)

Fig. 15

(continued) 


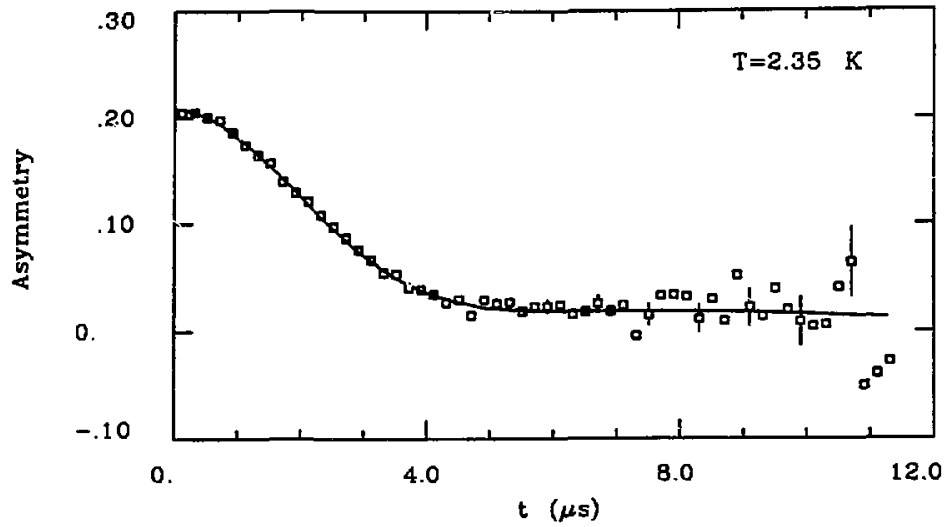

(c)

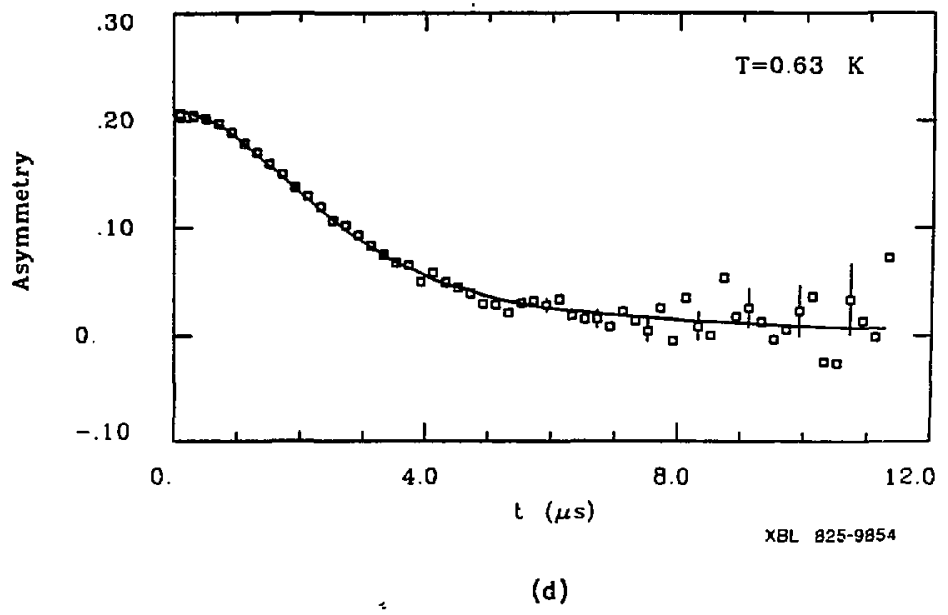

Fig. 15 


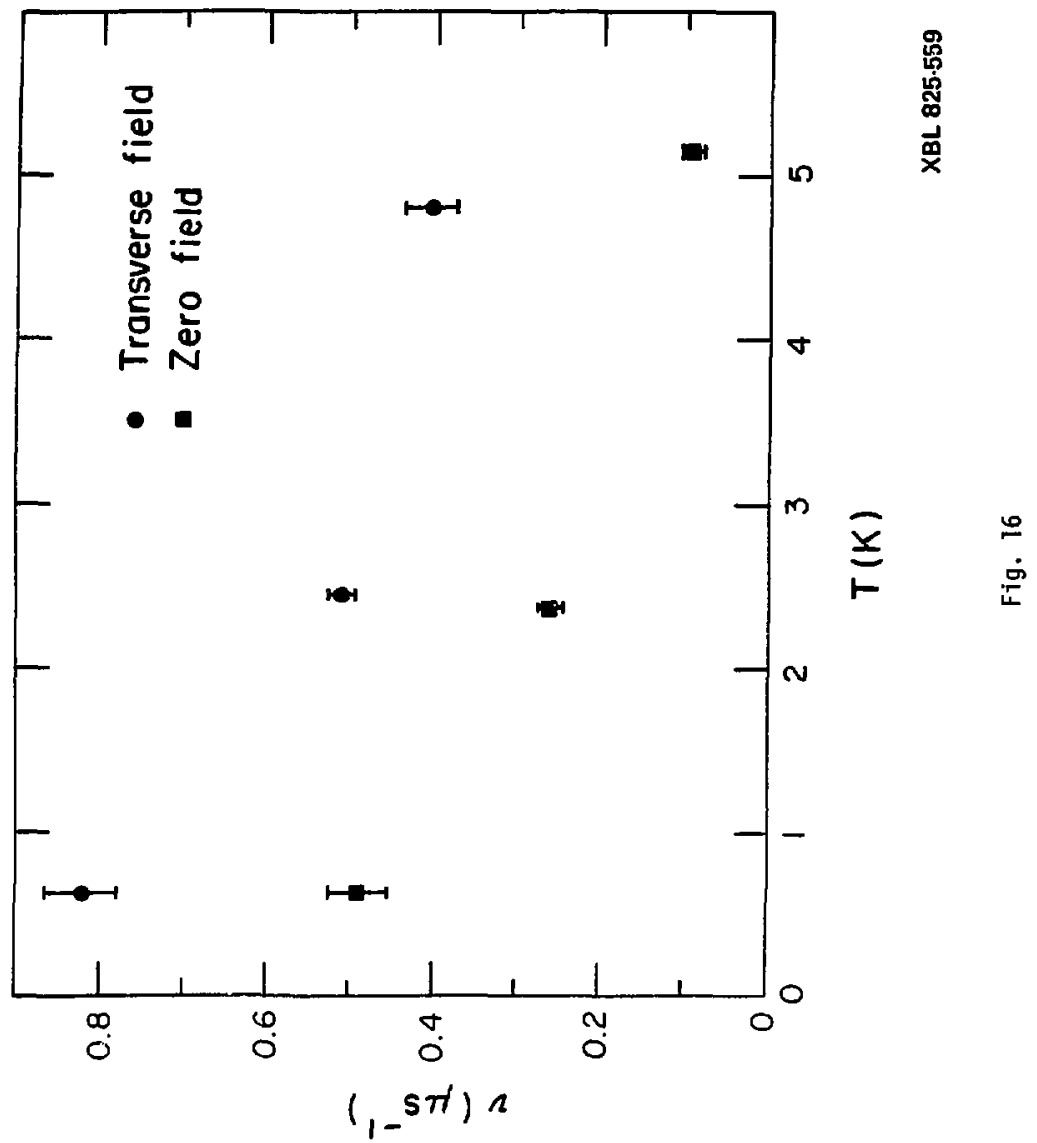




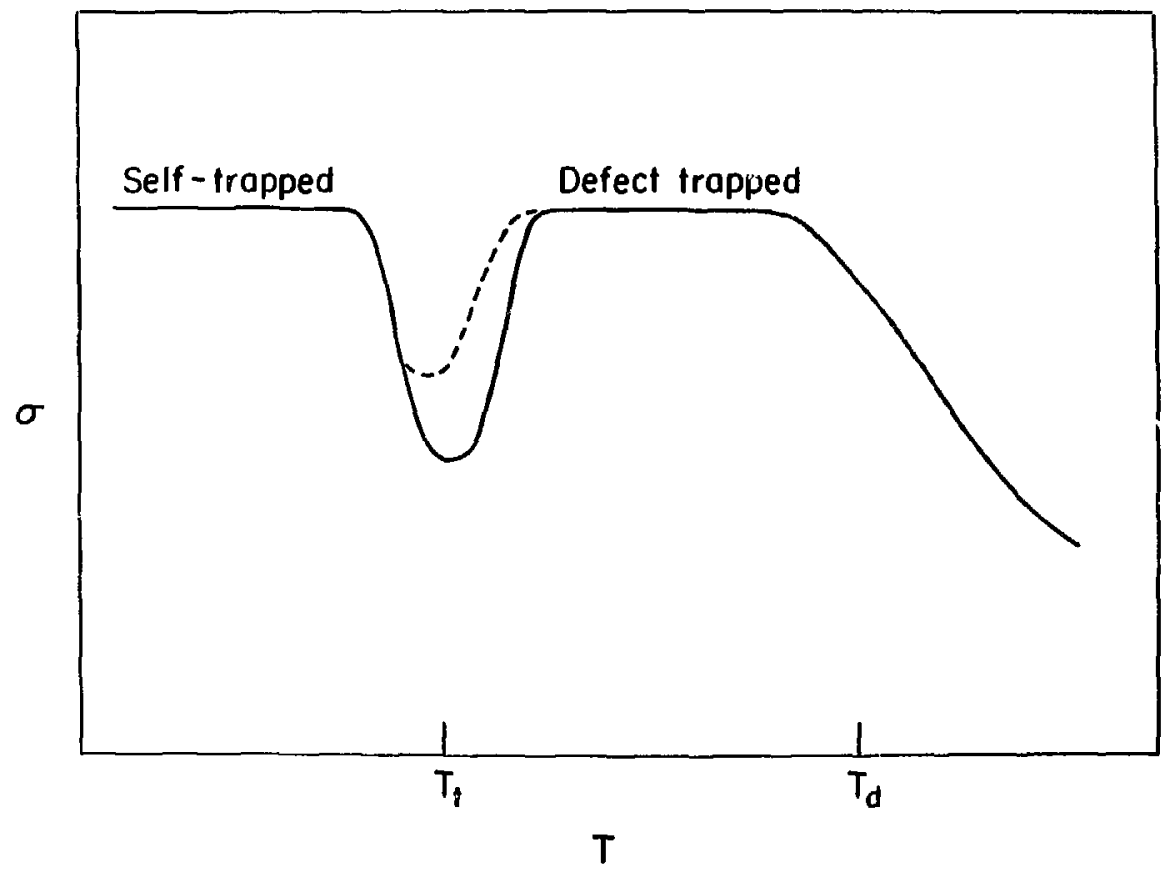

XBL 826-556

Fig. 17 


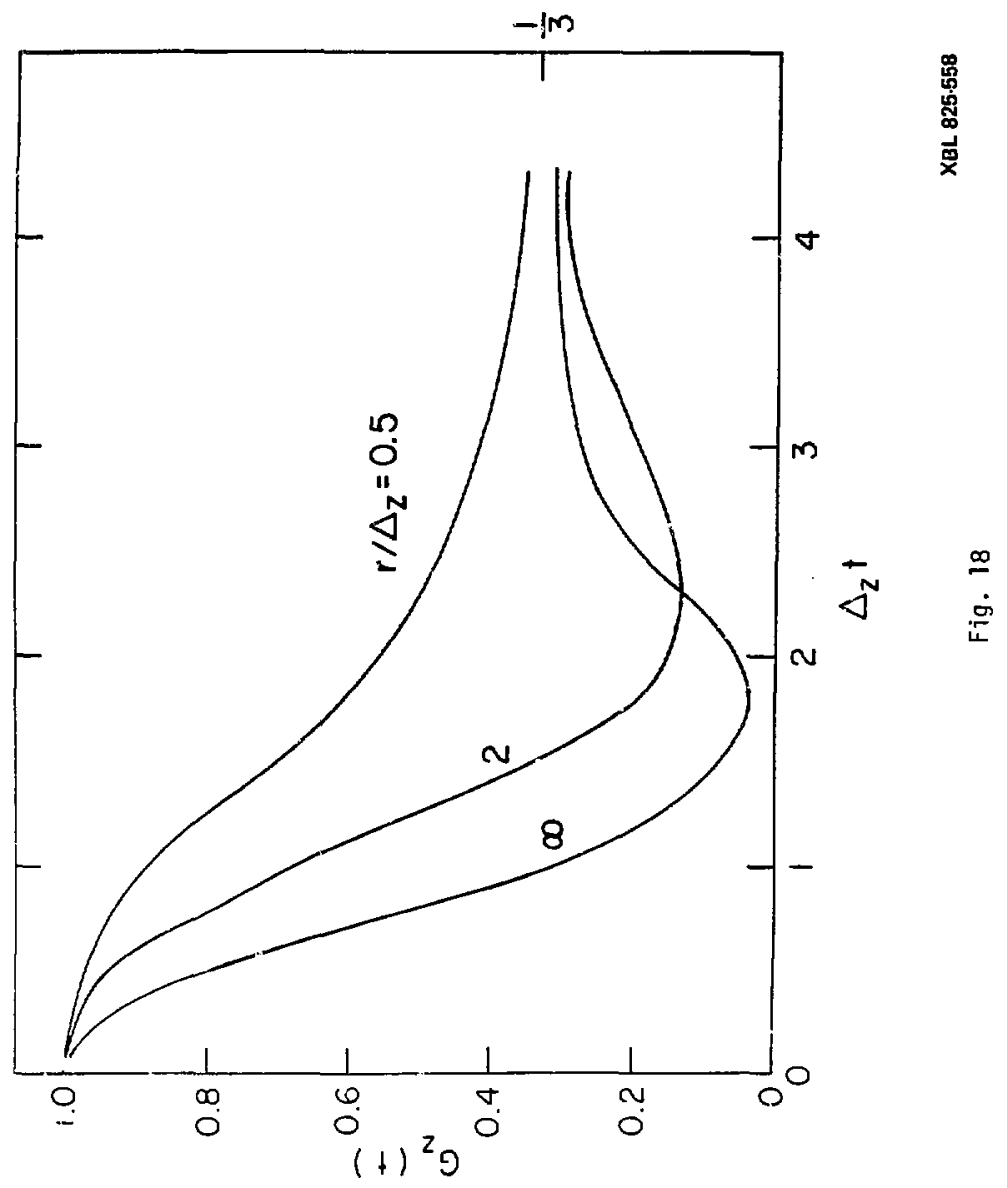


This reporl was done with support from the Department of Energy. Any conclusions or opinions expressed in this report represent solely thase of the author(s) and not necessarily those of The Regents of the University of California, the Lawrence Berkelty Laboratory or the Department of Energy.

Relerence to a company or product name does not imply approval or recommendation of the product by the University of California or the U.S. Department of Energy to the exclusion of others that may be suitable. 


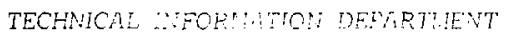

LAWRENCI GHREETEY LASORATRRY

UNTFESTY OF ARIFOPVI

BERKFLEY CHIFOFNA 9.170 